\title{
REVIEWS
}

\section{Engineering strategies to overcome the current roadblocks in CAR T cell therapy}

Sarwish Rafiq ${ }^{1,2}$, Christopher S. Hackett $\mathbb{B}^{3}$ and Renier J. Brentjens ${ }^{3,4,5 *}$

Abstract | T cells genetically engineered to express chimeric antigen receptors (CARs) have proven - and impressive - therapeutic activity in patients with certain subtypes of B cell leukaemia or lymphoma, with promising efficacy also demonstrated in patients with multiple myeloma. Nevertheless, various barriers restrict the efficacy and/or prevent the widespread use of CAR T cell therapies in these patients as well as in those with other cancers, particularly solid tumours. Key challenges relating to CAR T cells include severe toxicities, restricted trafficking to, infiltration into and activation within tumours, suboptimal persistence in vivo, antigen escape and heterogeneity, and manufacturing issues. The evolution of CAR designs beyond the conventional structures will be necessary to address these limitations and to expand the use of CAR T cells to a wider range of malignancies. Investigators are addressing the current obstacles with a wide range of engineering strategies in order to improve the safety, efficacy and applicability of this therapeutic modality. In this Review, we discuss the innovative designs of novel CAR T cell products that are being developed to increase and expand the clinical benefits of these treatments in patients with diverse cancers.

'Department of Hematology and Medical Oncology, Emory University School of Medicine, Atlanta, GA, USA.

${ }^{2}$ Winship Cancer Institute, Emory University, Atlanta $G A$, USA.

${ }^{3}$ Department of Medicine, Memorial Sloan Kettering Cancer Center, New York, $N Y$, USA.

${ }^{4}$ Cellular Therapeutics Center, Memorial Sloan Kettering Cancer Center, New York, $N Y$, USA.

${ }^{5}$ Molecular Pharmacology and Chemistry Program, Memorial Sloan Kettering Cancer Center, New York, $N Y$, USA.

*e-mail: brentjer@mskcc.org https://doi.org/10.1038/ s41571-019-0297-y
Chimeric antigen receptors (CARs) are synthetic receptors that redirect lymphocytes to recognize and eliminate cells expressing a cognate target ligand ${ }^{1}$. CAR T cells targeted at cancer cells are the most widely studied form of this technology. Following the demonstration of remarkable response rates in patients with large B cell lymphoma or acute lymphoblastic leukaemia $(\mathrm{ALL})^{2-5}$, adoptive transfer of autologous CD19-targeted CAR T cells became the first therapeutic approach with a genetic engineering component to be approved by the FDA for use in the USA ${ }^{6,7}$. Investigators are attempting to broaden the therapeutic benefits of CAR T cells by combining them with numerous other types of anticancer therapies (previously reviewed elsewhere ${ }^{8}$ ) or through innovations in CAR design to address the safety and production issues associated with the currently approved agents as well as to enhance their efficacy and overcome treatment resistance. In parallel, novel CAR engineering strategies are being developed to expand the clinical successes achieved with CAR T cells to patients with other malignancies, including solid cancers. In this Review, we provide an overview of basic CAR design and discuss emerging strategies to engineer safer and more effective CAR T cells for the treatment of haematological and solid cancers.

\section{CAR structure}

CARs have a modular design with four major components: an antigen-binding domain, a hinge, a transmembrane domain and an intracellular signalling domain (FIG. 1). Each of these elements has a distinct function and, optimal molecular design of the CAR can be achieved through many variations of the constituent protein domains.

\section{Antigen recognition and binding domains}

The antigen-binding domain is the extracellular portion of the CAR that recognizes the target antigen and redirects the specificity of CAR-expressing lymphocytes accordingly (FIG. 1). The antigen-binding domains of CARs have traditionally been composed of the variable heavy $\left(\mathrm{V}_{\mathrm{H}}\right)$ and variable light $\left(\mathrm{V}_{\mathrm{L}}\right)$ chains of monoclonal antibodies, connected by a flexible linker to form a singlechain variable fragment $(\mathrm{scFv})^{9}$. The $\left(\mathrm{Gly}_{4} \mathrm{Ser}\right)_{3}$ peptide is the most commonly used linker, exploiting glycine residues for flexibility and serine residues for solubility and resulting in a properly folded scFv capable of antigen recognition and binding ${ }^{10}$. CARs classically contain scFvs targeting extracellular antigens of cell-surface proteins expressed by cancer cells, thus enabling major histocompatibility complex (MHC)-independent $\mathrm{T}$ cell 


\section{Key points \\ - Chimeric antigen receptor (CAR) T cells have induced remarkable responses in patients with certain haematological malignancies, yet various barriers restrict the efficacy and/or prevent the widespread use of this treatment. \\ - Investigators are addressing these challenges with engineering strategies designed to improve the safety, efficacy and applicability of CAR T cell therapy. \\ - CARs have modular components, and therefore the optimal molecular design of the CAR can be achieved through many variations of the constituent protein domains. \\ - Toxicities currently associated with CAR T cell therapy can be mitigated using engineering strategies to make CAR T cells safer and that potentially broaden the range of tumour-associated antigens that can be targeted by overcoming on-target, off-tumour toxicities. \\ - CAR T cell efficacy can be enhanced by using engineering strategies to address the various challenges relating to the unique biology of diverse haematological and solid malignancies. \\ - Strategies to address the manufacturing challenges can lead to an improved CAR T cell product for all patients.}

Molecules other than $\mathrm{scFv}$ have been used as alternative antigen-binding domains for CARs. For example, zetakine CARs comprising cytokines fused to intracellular signalling domains, such as those targeting IL-13 receptor a2 (IL-13R 2 2) via membranetethered IL-13 linked to the intracellular 4-1BB and $\mathrm{CD} 3 \zeta$ domains, have been tested in clinical studies ${ }^{27}$. Other ligand-based CARs are being tested in preclinical and clinical studies across a range of malignancies, including those incorporating a proliferation-inducing ligand (APRIL) to target $\mathrm{B}$ cell maturation antigen (BCMA) and transmembrane activator and calciummodulator and cyclophilin ligand (TACI), which are receptors implicated in the pathogenesis of multiple myeloma ${ }^{28}$, FLT3 ligands to target FLT3 ${ }^{+}$acute myeloid leukaemia $(\mathrm{AML})^{29}$, granulocyte-macrophage colonystimulating factor (GM-CSF) to target the GM-CSF receptor (CD116) involved in the pathogenesis of juvenile myelomonocytic leukaemia ${ }^{30}$ or natural killer (NK) cell receptor D (NKG2D) to target NKG2D ligands on the surface of cancer cells ${ }^{31}$. Finally, T cells expressing CARs with peptide domains developed de novo for binding to specific antigens, such as designed ankyrin repeat proteins (DARPins) targeting HER2 (REFS ${ }^{32,33}$ ) or adnectin peptides (derived from tenth type III domain of human fibronectin) targeting $\mathrm{EFGR}^{34}$, have demonstrated preclinical efficacy.

\section{Hinge and transmembrane domains}

The hinge and transmembrane domains of CARs connect the extracellular antigen-binding domain to the intracellular signalling domains. The hinge provides sufficient flexibility to overcome steric hindrance and adequate length to facilitate access to the target antigen. Of note, differences in the length and composition of the hinge can affect antigen binding and signalling through the $\mathrm{CAR}^{35}$. The characteristics of the hinge and transmembrane domain also influence CAR T cell cytokine production and $\mathrm{AICD}^{36}$. Spacer sequences in the hinge domain enable the CAR to access membrane proximal antigen epitopes ${ }^{37-40}$, albeit at the potential cost of decreased CAR T cell function ${ }^{41,42}$. Amino acid sequences from CD8, CD28, IgG1 or IgG4 have been utilized in CAR hinge domains (FIG. 1), although some of the IgG-derived peptides can interact with Fc $\gamma$ receptors $(\mathrm{Fc} \gamma \mathrm{Rs})$, leading to CAR T cell depletion and thus decreased persistence in vivo ${ }^{43,44}$.

The transmembrane domain anchors the CAR in the $\mathrm{T}$ cell membrane and is usually derived from type I proteins such as $\mathrm{CD} 3 \zeta, \mathrm{CD} 28, \mathrm{CD} 4$ or CD8a (FIG. 1). The transmembrane domain used influences the stability and function of the $\mathrm{CAR}^{45,46}$. For example, CARs containing the CD28 transmembrane domain are more stable than those harbouring the transmembrane region of $\mathrm{CD} 3 \zeta^{47}$. Interestingly, however, the $\mathrm{CD} 3 \zeta$ transmembrane domain mediates CAR dimerization and incorporation into endogenous TCRs, which might facilitate CARmediated T cell activation ${ }^{45}$. CAR T cells with CD8a hinge and transmembrane domains have been shown to release less IFN $\gamma$ and TNF and are less susceptible to AICD than those in which these domains are derived from CD28 $\left(\right.$ REF. $\left.^{36}\right)$. 


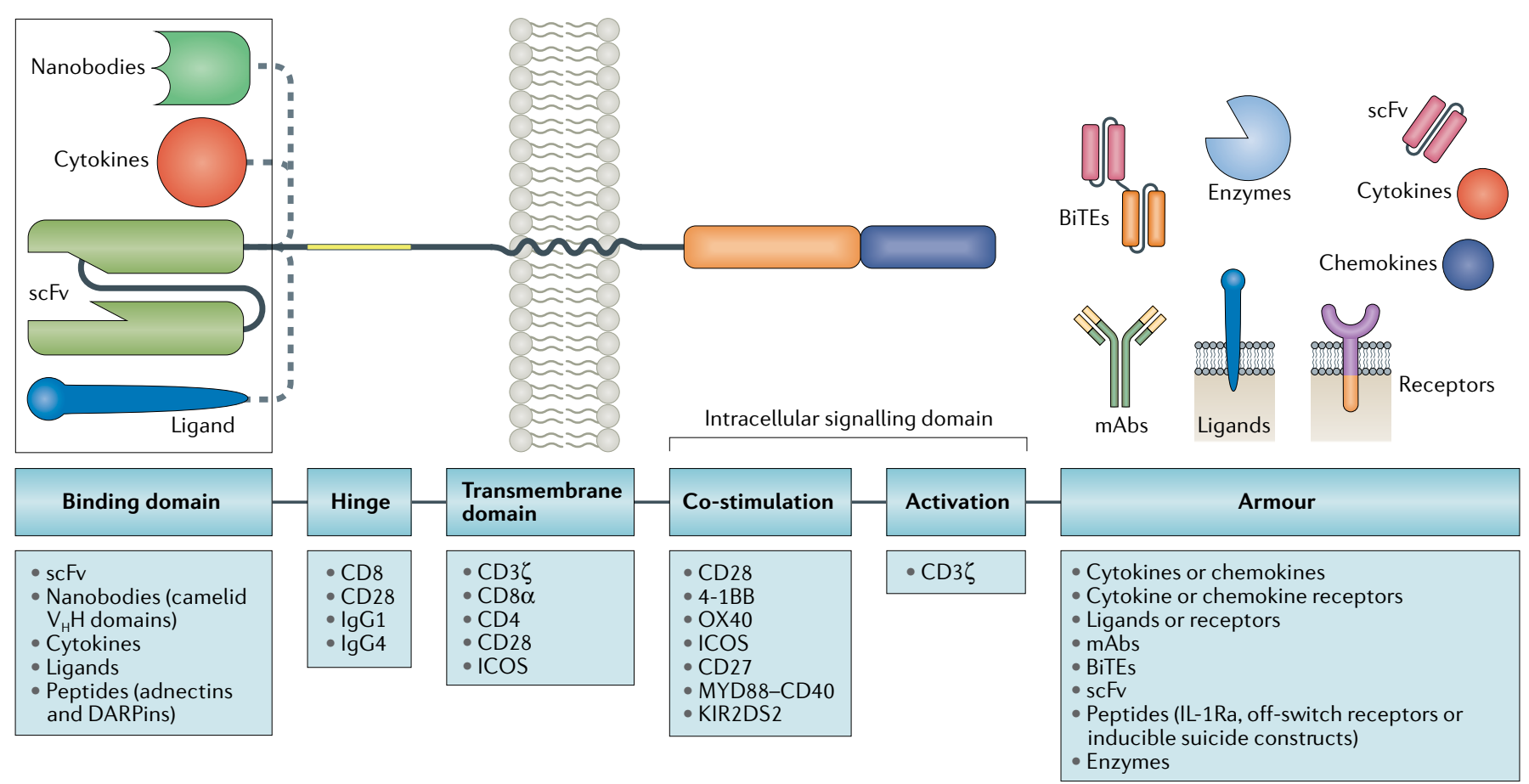

Fig. 1 | Blueprint of CAR design. Chimeric antigen receptors (CARs) have a modular design consisting of an antigenbinding domain, a hinge, a transmembrane domain and an intracellular signalling domain. In preclinical and clinical studies of CAR T cells, investigators have used reference sequences from a myriad of molecules within each of these domains. The antigen-binding domain is usually a single-chain variable fragment (scFv) molecule derived from a monoclonal antibody (mAb; from mouse anti-human CD19 antibodies, for example, in the currently FDA-approved CAR T cell products). The intracellular signalling domain generally contains a T cell activation domain derived from the CD3 $\zeta$ chain of the T cell receptor as well as co-stimulatory domains that often comprise immunoreceptor tyrosine-based activation motif-containing regions of CD28 or 4-1BB (also known as CD137 and TNFRSF9). CAR gene constructs can be further modified to engineer CAR T cells with expression of an 'armour' protein, which is typically a cell-surface or secreted immunomodulatory molecule that enhances $T$ cell function or favourably modifies the tumour microenvironment. Variation of each of these component parts of CAR constructs enables fine tuning of the functionality and antitumour activity of the resultant CAR T cell product, and CARs with various designs are being developed to improve the safety and efficacy of these therapies across various cancers. In addition, gene editing of the engineered T cells to further enhance CAR T cell function is a promising avenue of research in this area. BiTEs, bi-specific T cell engagers; DARPins, designed ankyrin repeat proteins; ICOS, inducible T cell co-stimulator; IL-1Ra, IL-1 receptor antagonist; KIR2DS2, killer cell immunoglobulin-like receptor 2DS2; $\mathrm{V}_{\mathrm{H}} \mathrm{H}$, variable domain of a heavy chain antibody.

\section{Intracellular signalling domains}

The fourth component of the CAR, the intracellular signalling domain, typically comprises an activation domain and one or more co-stimulatory domains (FIG. 1). The vast majority of CARs activate CAR T cells via $\mathrm{CD} 3 \zeta$ derived immunoreceptor tyrosine-based activation motifs. However, signalling mediated by these motifs alone is insufficient to induce productive $\mathrm{T}$ cell responses and results in limited in vivo $\mathrm{T}$ cell persistence and activity $^{48}$; a co-stimulatory signal is necessary for optimal T cell function, metabolism and persistence. T cells with CARs containing co-stimulatory domains in addition to activation domains produce IL- 2 and can proliferate upon repeated antigen exposure ${ }^{49}$. The most widely studied co-stimulatory domains are derived from CD28 or 4-1BB (CD137), and CAR T cell products utilizing either of these domains are FDA approved ${ }^{6,7}$. CD28-domain CAR T cells and 4-1BB-domain CAR T cells are both associated with high response rates in patients but have different functional and metabolic profiles ${ }^{50}$. T cells expressing CARs with CD28 domains differentiate into effector memory T cells and predominantly utilize aerobic glycolysis, whereas 4-1BB-containing CAR T cells differentiate into central memory $\mathrm{T}\left(\mathrm{T}_{\mathrm{CM}}\right)$ cells and have increased mitochondrial biogenesis and oxidative metabolism ${ }^{51}$. T cells with CARs containing alternative co-stimulatory domains have demonstrated efficacy in preclinical models but have not yet been tested in patients. These domains include those derived from MYD88 and CD40 (REF. ${ }^{52}$ ), OX40 (CD134) ${ }^{53,54}$, inducible T cell co-stimulator $(\mathrm{ICOS})^{55}, \mathrm{CD} 27\left(\mathrm{REFS}^{56,57}\right)$ and killer cell immunoglobulin-like receptor 2DS2 (KIR2DS2; combined with co-expression of TYRO protein tyrosine kinase-binding protein, also known as DAP12) $)^{58}$.

Building on the four modular components of CARs and informed by the success and failures of CAR T cells in clinical studies, investigators are innovatively engineering CAR T cells in a variety of ways. These strategies aim to improve the safety and efficacy of CAR T cell therapies, broaden the range of cancers amenable to such treatments and facilitate more rapid, reliable and efficient production of these agents. 


\section{Overcoming treatment-related toxicities}

Therapeutic responses to CAR T cells in patients who otherwise have limited treatment options have been dramatic in some trials ${ }^{2-5}$, although at the cost of high rates of severe adverse events, including some fatalities. These toxicities have been best characterized in patients treated with CD19-directed CAR T cells, which were among the earliest CAR T cell therapies used in successful clinical trials and the first to gain FDA approval ${ }^{6,7}$. Mechanistically, the major CAR T cell toxicities can be divided into two categories: 1) general toxicities related to $\mathrm{T}$ cell activation and subsequent systemic release of high levels of cytokines; and 2) toxicities resulting from specific interactions between the CAR and its target antigen expressed by non-malignant cells - termed on-target, off-tumour effects.

\section{Systemic cytokine toxicities}

Severe and sometimes lethal increases in systemic cytokine levels have been observed in patients treated with CAR T cells in many clinical trials, including all of the successful trials of anti-CD19 CAR T cells ${ }^{2-5}$. These effects reflect the robust interactions of CAR T cells with cancer cells and/or cells of the host immune system, which result in CAR T cell activation and expansion and, in some patients, a vicious cycle of immune cell cross-activation and systemic cytokine release that can reach toxic levels ${ }^{59}$. These toxicities include cytokine-release syndrome (CRS), haemophagocytic lymphohistiocytosis (HLH) and/or macrophage activation syndrome (MAS), and a distinct neurotoxicity now referred to as immune effector cell-associated neurotoxicity syndrome (ICANS) ${ }^{60}$.

CRS is characterized by increased serum levels of inflammatory cytokines, fever, hypotension, hypoxia and organ dysfunction and is often responsive to treatment with the anti-IL- 6 receptor antibody tocilizumab, the anti-IL-6 antibody siltuximab or corticosteroids. CAR T cell-induced HLH/MAS is a rare and more severe systemic hyperinflammatory syndrome diagnosed based on manifest symptoms of CRS and combinations of elevated serum levels of ferritin and liver enzymes, haemophagocytosis, cytopenias, renal failure, pulmonary oedema, splenomegaly and/or an absence of NK cell activity. CAR T cell-related HLH/MAS can be refractory to targeted inhibition of the IL-6 pathway and might require the use of chemotherapy (systemic etoposide or, in the context of HLH-associated neurotoxicity, intrathecal cytarabine), extrapolating from the management paradigms of non-CAR-related refractory $\mathrm{HLH}^{2}$.

ICANS is associated with disruption of the bloodbrain barrier and increased cerebrospinal fluid cytokine levels ${ }^{61}$ and can present as aphasia, an altered mental state, tremor, seizures, headache and life-threatening cerebral oedema, often occurring concurrently with or following $\mathrm{CRS}^{60}$. Management of ICANS can involve corticosteroids and/or IL-6 pathway inhibitors if symptoms of CRS are also present as well as appropriate adjunctive and/or supportive treatment of neurological symptoms and abnormalities evident on neuroimaging and electroencephalography ${ }^{2}$.
Increased appreciation and understanding of these characteristic toxicities has led to improved clinical management, with the establishment of grading and treatment guidelines ${ }^{2,60}$, changes to the method of cell isolation during manufacturing ${ }^{62}$ and altered dosing levels ${ }^{3,62}$. Agents targeting the IL-6 pathway, such as tocilizumab or siltuximab, are increasingly used in addition to, or as a replacement for, corticosteroids, thus reducing or avoiding the observed detrimental effects of corticosteroids on the antitumour activity of CAR T cells ${ }^{63}$. The risk of cytokine-related toxicities is proportional to tumour burden and can, therefore, be attenuated by paradoxically decreasing the number of cells infused in patients with high-volume disease; however, this association is inexact ${ }^{3,61}$, and fundamental modifications of CAR designs might be a better approach to ameliorate cytokine-related toxicities.

To achieve clinical efficacy while avoiding general systemic cytokine toxicities, CAR T cells must reach a threshold level of activation and cytokine secretion without exceeding a level that results in a vicious cycle of cytokine release. The degree and kinetics of CAR T cell activation is influenced by the overall tumour burden, the level of antigen expression on the tumour cells, the affinity of the scFv (or other antigen-binding domain) for the antigen and the co-stimulatory elements included in the CAR, along with other factors ${ }^{64,65}$. Thus, the therapeutic window of CAR T cell activation will be different with different CARs and will need to be established for each CAR using careful dose-escalation schedules in phase I trials. Nevertheless, several components of the CAR can be engineered to optimize this therapeutic window.

CD28 co-stimulatory domains have been associated with a more rapid onset of CAR T cell activity and subsequent exhaustion than 4-1BB domains; correspondingly, 4-1BB domains have been associated with lower peak levels of $\mathrm{T}$ cell expansion, leading to increased $\mathrm{T}$ cell endurance and a lower risk of cytokine-mediated toxicities $^{66}$. Thus, the choice of co-stimulatory domain offers a modifiable variable in CAR design that can potentially be tailored to the tumour type and burden, the target antigen and antigen density, and the specific CAR scFv used. Inclusion of a 4-1BB co-stimulatory domain might be sufficient and less toxic in patients with a high disease burden and/or with tumours with a high antigen density (resulting in a high CAR scFv avidity); however, a CD28 domain might be needed to reach the required threshold of $\mathrm{T}$ cell activation in the context of a low total surface antigen density and/or a CAR with a low-affinity antigen-binding domain.

Cytokine secretion by activated CAR T cells can also be modulated through engineering of other parts of the CAR construct. For example, modification of the CD8aderived hinge and transmembrane amino acid sequences of a CD19-targeted CAR resulted in reduced CAR T cell proliferation and lower levels of cytokine release ${ }^{67}$. In a phase I trial ${ }^{67}$, these CAR T cells induced complete remissions in 6 of 11 (54.5\%) patients with B cell lymphoma, with no grade $>1$ ICANS or CRS observed.

In addition to the cytokine storm induced by cytokines released from activated CAR T cells, recognition of the CAR constructs by host immune cells and the subsequent 
immune responses might also contribute to cytokinerelated toxicities. The use of human or humanized antibody fragments for CAR construction ${ }^{68}$, rather than those derived from mouse antibodies, as well as modification of the extracellular hinge region and/or transmembrane domain $^{43,44}$ can reduce the immunogenicity of the CAR. Importantly, this strategy might simultaneously decrease the risk of cytokine-mediated toxicities and improve CAR T cell persistence ${ }^{44,68}$.

The $\mathrm{T}$ cells transduced with the CAR can also be further modified through genetic engineering in order to avert or ameliorate toxicities. In preclinical models, inhibition of the macrophage-activating and monocyteactivating cytokine GM-CSF using the antibody lenzilumab increases CAR T cell activity while decreasing the risk of CRS, and mutational inactivation of GM-CSF in the transduced T cells has similar effects ${ }^{69,70}$. Similarly, preclinical evidence implicates IL-1 released by monocytes and/or macrophages in CAR T cell-associated CRS and ICANS ${ }^{71,72}$. Accordingly, IL-1 receptor antagonists, either administered systemically or secreted from engineered CAR T cells, ameliorate CRS and neurotoxicity in mouse models of leukaemia or lymphoma treated with anti-CD19 CAR T cells $\mathrm{s}^{71,72}$. In addition, myeloid cell-specific deletion of tyrosine hydroxylase or inhibition of this enzyme using metyrosine reduces circulating catecholamine and, in turn, cytokine levels after antiCD19 CAR T cell therapy in a mouse model of lymphoma $^{73}$. Further modification of these pathways through CAR T cell engineering could provide opportunities to reduce the toxicity of these agents. Alternatively, CARs can be engineered to recognize bi-specific adapter molecules that also contain moieties that bind to receptors expressed on cancer cells (for example, folate receptors), thus bridging CAR T cells and cancer cells (FIG. 2Aa); dosing of these short half-life, small-molecule adaptors can then be controlled dynamically in order prevent or terminate $\mathrm{CRS}^{74,75}$.

Another strategy to control the toxicity of CAR T cells is the engineering of 'off switches' or 'suicide genes' into the CAR construct, providing a means to deactivate CAR T cells if and when either cytokine-mediated or on-target, off-tumour toxicities occur (FICS 2Ab-d, 2Ba,b). Numerous approaches have been developed, some based on co-expression of the CAR and human cellsurface antigens for which FDA-approved therapeutic antibodies already exist (FIG. 2Bb), including CD20, which is targetable with rituximab ${ }^{76-78}$, or the extracellular and transmembrane domains of EGFR, which can be targeted with cetuximab ${ }^{79}$. Administration of rituximab or cetuximab to patients with CAR T cell-related toxicities has not been reported and thus the clinical efficacy of this approach remains to be determined. Notwithstanding, a limitation of this approach is the slow onset of antibody-mediated killing of CAR T cells, which might limit its utility in patients with severe, acute cytokine-mediated toxicities that require rapid reversal.

A more rapid safety switch has been developed using an apoptosis-triggering fusion protein comprising caspase 9 linked to a modified form of the FK506binding protein FKBP1A (iCasp9), with the latter enabling conditional dimerization and activation of the fusion protein through binding to a systemically administered and otherwise biologically inert small molecule (AP1903) (FIG. 2Ba). In a clinical trial, five patients received an allogeneic haematopoietic stem cell transplant for the treatment of acute leukaemia followed by iCasp9-modified donor T cells to enhance immune reconstitution; the dimerizing agent was subsequently administered to four patients who developed graft versus host disease (GVHD), resulting in elimination of $>90 \%$ of the modified T cells within 30 minutes and resolution of $\mathrm{GVHD}^{80}$, thus demonstrating the potential of this approach for rapid depletion of CAR T cells.

Another strategy to dynamically and rapidly control CAR T cell function involves the regulation of the expression of CARs on the surface of T cells using the proteasebased small molecule-assisted shutoff CARs (SMAShCARs), also known as switch-off CARs (SWIFF-CARs) ${ }^{81}$ (FIG. 2Ad). In this system, a protease target site and protease are embedded in the CAR construct, together with a 'degron' moiety that promotes degradation of the CAR protein. In the 'on' state, the target site is cleaved, resulting in removal of the degron from the CAR protein, and thus the CAR is expressed on the surface of the T cells; however, upon administration of an exogenous smallmolecule protease inhibitor, the CAR protein is not cleaved, resulting in retention of the degron and CAR degradation via the proteolytic pathway.

More recently, Mestermann et al. ${ }^{82}$ demonstrated that the tyrosine kinase inhibitor dasatinib, which is an FDAapproved treatment of Philadelphia chromosomepositive chronic myeloid leukaemia and ALL, interferes with CAR activity by inhibiting LCK, thereby disrupting signalling downstream of the activated $\mathrm{CD} 3 \zeta$ domain (FIG. 2AC). Accordingly, dasatinib rapidly and reversibly prevented CAR T cell activation and, when administered shortly (3 hours) after cell infusion, markedly reduced mortality from CAR T cell-induced CRS in a mouse model of lymphoma ${ }^{82}$. If these observations are validated in clinical studies, dasatinib could provide a readily available, reversible and well-tolerated pharmacological safety switch that, unlike many other safety switches, does not result in eradication of CAR T cells.

Thus, safety switches hold promise as a means of eliminating CAR T cells when toxicities arise; however, in clinical practice, CAR T cells are generally sensitive to corticosteroids and chemotherapeutic agents (such as cytarabine and fludarabine) ${ }^{59}$, which provide well-established and widely available backstops should these safety switches fail.

\section{On-target, off-tumour toxicity}

Successful use of CAR T cells in the treatment of patients with B cell malignancies has been associated with B cell aplasia and resultant hypogammaglobulinaemia owing to CAR T cell-mediated eradication of $\mathrm{CD} 19^{+} \mathrm{B}$ cell progenitors in the bone marrow ${ }^{83}$. This widely observed toxicity of anti-CD19 CAR T cells is well tolerated and treatable with periodic infusions of intravenous immunoglobulins to replace antibodies that would otherwise have been produced by the patient's B cells. The potential for less tolerable on-target, off-tumour toxicities with agents targeting other candidate TAAs limits the clinical use of 
A 'On/off switches' predicated on administration of small-molecule agents

a

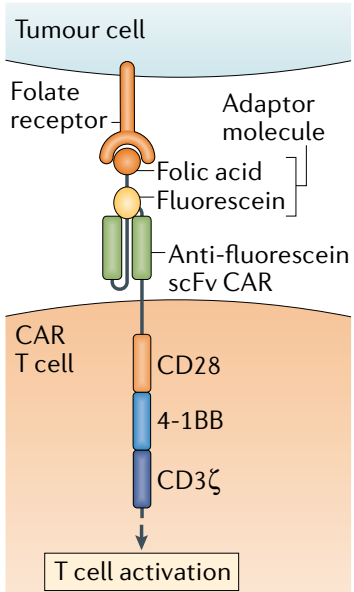

B Suicide gene systems

a

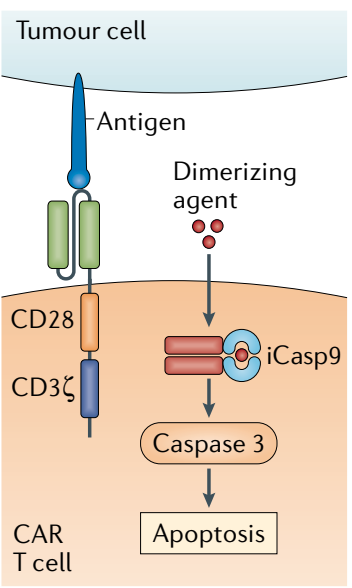

b

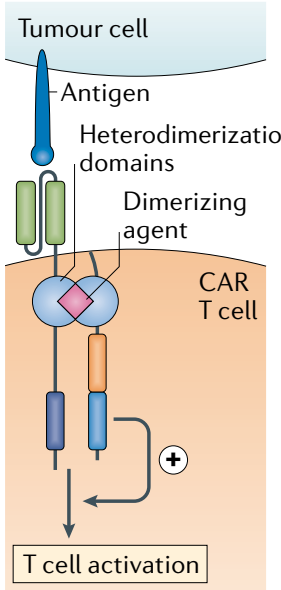

b

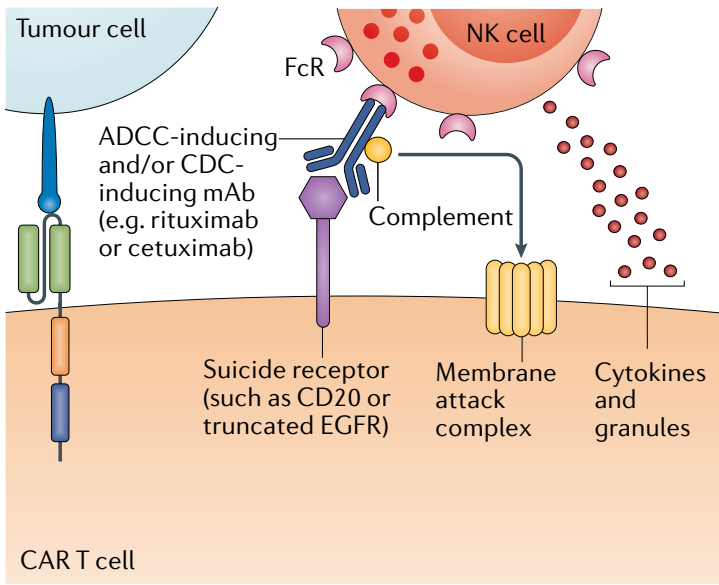

ON

d
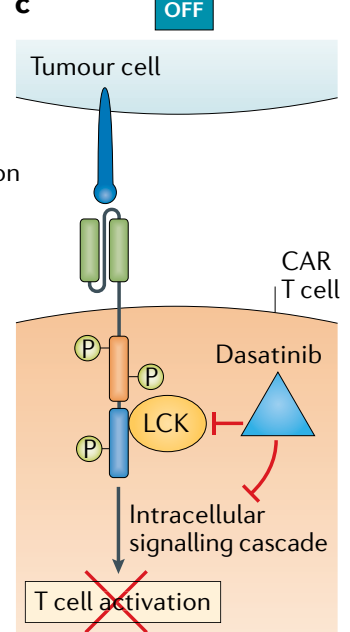

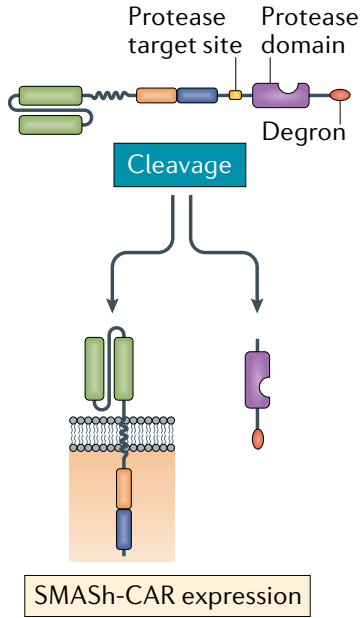

OFF
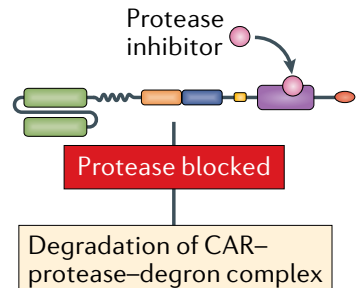

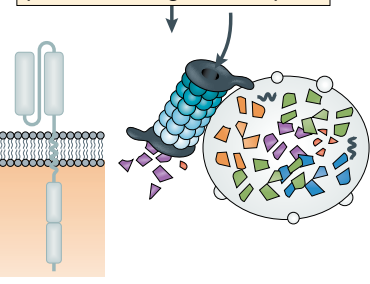

SMASh-CAR not expressed

C Direct antagonism of systemic cytokines

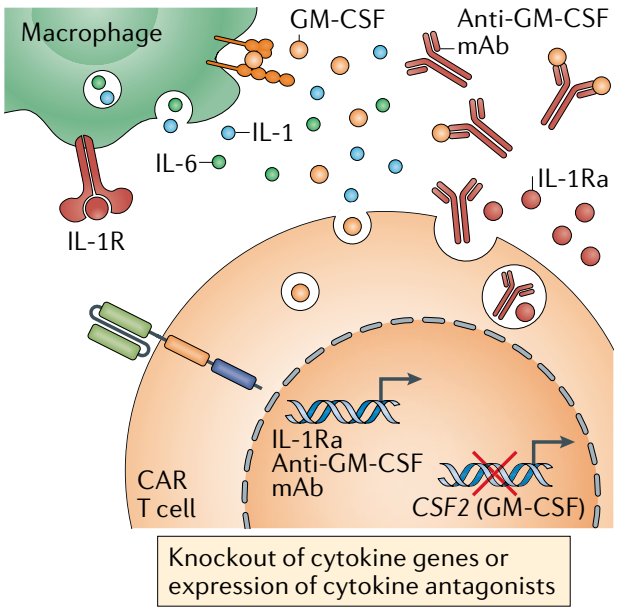

Fig. 2 | Overcoming systemic cytokine toxicities of CAR T cells. The activation and rapid expansion of chimeric antigen receptor (CAR) T cells in patients treated with these agents is associated with high systemic levels of cytokines. To counter this effect in the event of systemic cytokine-related toxicities, researchers are engineering methods to control CAR expression or activity. A | CAR T cells with on/off switches predicated on small-molecule adapter ligands (a), CAR subunitdimerizing agents (b), inhibitors of signalling downstream of the CAR (c), or protease inhibitors used to control CAR protein expression (d). B | Suicide gene systems that enable elimination of CAR T cells via induction of apoptosis (a) or antibodydependent cellular cytotoxicity (ADCC) and/or complement-dependent cytotoxicity (CDC) (b). C $\mid C A R T$ cells engendered with the intrinsic ability to secrete factors that neutralize relevant cytokines. FcR, fragment crystallizable region receptor; GM-CSF, granulocyte-macrophage colony-stimulating factor; iCasp9, inducible caspase 9; IL-1R, IL-1 receptor; IL-1Ra, IL-1 receptor antagonist; mAb, monoclonal antibody; NK, natural killer; scFv, single-chain Fv; SMASh-CAR, small molecule-assisted shutoff chimeric antigen receptor.

many CAR T cell products. In a clinical trial of a carbonic anhydrase IX (CAIX)-targeted CAR T cell product involving patients with renal cell carcinoma, multiple patients developed liver enzyme abnormalities ${ }^{84}$. These adverse effects were attributed to CAR T cell infiltration into the CAIX-expressing bile duct epithelium and were prevented in subsequent patients through the administration of an antagonistic anti-CAIX monoclonal antibody ${ }^{84}$, providing strong evidence of on-target, off-tumour toxicities. Much more alarmingly, a patient receiving HER2-directed CAR T cell therapy for metastatic colon cancer developed respiratory distress and pulmonary oedema 15 minutes after cell infusion, which progressed to multiorgan failure and death ${ }^{85}$. The mechanism of this toxicity was speculated to be mediated by recognition of HER2 in non-malignant lung tissue by CAR T cells, triggering a systemic cytokine storm ${ }^{85}$. Of note, HER2 is expressed at low levels in many nonmalignant tissues, and the antibody trastuzumab, on which the CAR was based, has known on-target, offtumour effects ${ }^{86}$. All other elements of the trial protocol, including the history of agents targeting HER2, the type of retrovirus used to generate CAR T cells and the $\mathrm{T}$ cell dose, were determined based on clinical experience to be safe ${ }^{85}$, supporting the evidence suggesting that CAR T cell responses to HER2 expressed on 
non-malignant lung cells caused the toxicity. However, the onset of symptoms only 15 minutes after infusion is uncharacteristic of $\mathrm{T}$ cell-mediated responses. Moreover, the clinical presentation resembled acute respiratory distress syndrome ${ }^{85}$, which often occurs as a result of inflammation from a source outside of the lungs (for example, the bloodstream or pancreas, or as a sequelae of transfusion-related lung injury), and therefore the cytokine storm could potentially have resulted from an immune process unrelated to the CAR interacting with HER2 in lung tissue. HER2-targeted CARs used in trials involving patients with sarcoma have been better tolerated, with no reported CRS or ICANS ${ }^{87}$. Nevertheless, similar episodes of pulmonary oedema, including one fatal event, have occurred in patients with glioma after treatment with a CAR T cell product targeting EGFR variant III (EGFRvIII) ${ }^{88}$, although, again, without definitive evidence of a specific interaction of the CAR with EGFR expressed on non-malignant lung cells. Less severe pulmonary toxicities have also been observed in a trial of CAR T cells targeting carcinoembryonic antigen-related cell adhesion molecule 5 (CEACAM5), specifically in all of four patients who received a high CAR T cell dose (a total of $0.33-3.89 \times 10^{9}$ viable cells) and intensive preconditioning with cyclophosphamide and fludarabine ${ }^{89}$. These toxicities were hypothesized to be attributable to interactions between CAR T cells and non-malignant CEACAM5-expressing cells in the lung; however, no definitive evidence of such interactions was established and no gastrointestinal toxicities were observed ${ }^{89}$, despite the known expression of CEACAM5 in the gastrointestinal tract. Considering the variations in co-stimulatory domains, conditioning regimens, cytokine administration and CAR T cell dosing used in these studies, and that some of these toxicities are hard to distinguish from general cytokine-related toxicities, establishing whether the clinical complications reflect on-target, off-tumour effects is difficult. Nonetheless, technologies designed to limit on-target, off-tumour toxicities could provide opportunities to generate more clinically effective CAR therapies, and numerous approaches are currently in development ${ }^{90}$. These strategies are predicated on the targeting of antigens more specific to the tumour, optimizing the interaction of CARs with cancer cells relative to non-malignant cells (FIG. $3 \mathrm{Aa}-\mathrm{d}$ ), introducing the requirement for multiple antigens or the absence of a specific antigen (logic-gated CARs) (FIC. 3Ba) and/or limiting the spatial and temporal activity of CARs (FIG. 3Bb).

If tumour-specific antigens cannot be identified, another strategy to target cancer cells and avoid the toxic effects on non-malignant tissues that also express the target antigen relies on differences in antigen expression levels between tumour and non-malignant tissues - an approach termed affinity tuning. Antigens expressed at higher levels on tumour cells than on non-malignant cells could potentially be targeted using lower-affinity scFv CARs, thereby ensuring that only the tumour cells with a high antigen density can provide interactions of sufficiently high avidity to activate CAR T cells. The feasibility of this approach has been demonstrated in preclinical models, across a range of different antigens, although downregulation of the target antigen has been identified as a mechanism of resistance ${ }^{23,91,92}$. In one preclinical study in which the affinity of a ganglioside GD2-targeted CAR was dramatically increased through a single amino acid substitution in the antigen-binding domain $^{93}$, mice developed fatal encephalitis attributed to the interaction of CAR T cells with GD2 ${ }^{+}$cerebellar tissue that has not been observed with the lower-affinity GD2 CAR in preclinical models or clinical trials. This finding illustrates the profound effects that subtle changes in the scFv can have on the therapeutic window of CAR T cells.

Synthetic biology paradigms have also been used to increase the specificity of CAR T cells for tumour cells based on the detection of multiple antigens in order to overcome the lack of unique tumour-specific antigens. The synNotch system ${ }^{94}$ involves the use of a synthetic Notch receptor engineered to recognize one TAA, activation of which induces the expression of a second construct encoding a CAR specific to a second TAA and containing T cell activation domains - thus creating an "AND" logic gate requiring the simultaneous presence of two cell-surface antigens for CAR T cell activation (FIG. 3Ba). This system has been used to target tumour cells expressing the antigen ROR1 (REF. ${ }^{95}$ ), which is also present on essential bone marrow stromal cells and in other tissues; gating for expression of epithelial cell adhesion molecule (EpCAM) or B7-H3 as secondary antigens was demonstrated to increase the tumour-cell specificity and reduce the risk of toxicities in animal models, compared with traditional CAR T cells targeting ROR1 alone ${ }^{95}$. However, the slow activation kinetics of such logic-gated CAR T cells might limit the efficacy of this approach. A similar logic-gating effect can be achieved by linking the $\mathrm{CD} 3 \zeta$ and co-stimulatory domains to separate receptors, each recognizing different antigens (thus utilizing a first-generation CAR construct in combination with a chimeric co-stimulatory receptor) (FIG. 3Aa). The promise of this approach has been demonstrated in preclinical models of prostate cancer ${ }^{96}$, breast cancer $^{97}$ and other experimental systems ${ }^{98}$. An analogous approach involves the use of secondary chimeric cytokine receptors for tumour cell-derived cytokines, such as IL-4, to restrict T cell activity to tumour tissue ${ }^{90,99}$ (FIG. 3Ad), although with the drawback that these inflammatory cytokines are not strictly tumour specific. A related strategy is predicated on the use of inhibitory CARs, which are engineered to inhibit $\mathrm{T}$ cell activation upon binding to an antigen expressed on non-malignant cells but not tumour cells, thus ensuring that productive signalling by a co-expressed activatory CAR only occurs in tumours. Instead of co-stimulatory domains, the inhibitory CARs contain inhibitory domains derived from immune-checkpoint proteins, such as cytotoxic lymphocyte protein 4 (CTLA-4) or programmed cell death 1 (PD-1), thereby co-opting the intrinsic immune-checkpoint signalling pathways of T cells $^{100}$ (FIG. 3Ab).

Numerous approaches have been developed to limit the spatial distribution and duration of CAR T cell activity in order to restrict systemic off-tumour toxicities. For example, a system exploiting the hypoxia-inducible factor 1 a degradation pathway has been used with the aim of restricting CAR expression to only those CAR T cells located in the hypoxic $\mathrm{TME}^{101}$, thereby reducing the 
adverse effects on non-malignant tissues (which are largely non-hypoxic) (FIG. 3Bb). Transient expression of CARs can be achieved by using mRNA rather than integrating DNA vectors to transduce $T$ cells with the CAR construct ${ }^{21,102}$. Potentially toxic CAR T cell products can also be controlled using various 'on-switches', including pharmacological agents that mediate transient activating structural changes of the CAR constructs such as dimerization of different peptide chains containing dissociated, trans-acting activatory and co-stimulatory domains $^{103,104}$ (FIG. 2Ab).

An alternative approach to attenuating the innate toxic potential of conventional CAR T cells involves the introduction of the CARs into a $\mathrm{T}$ cell subset with more
A Improving specificity through targeting of multiple antigens

a
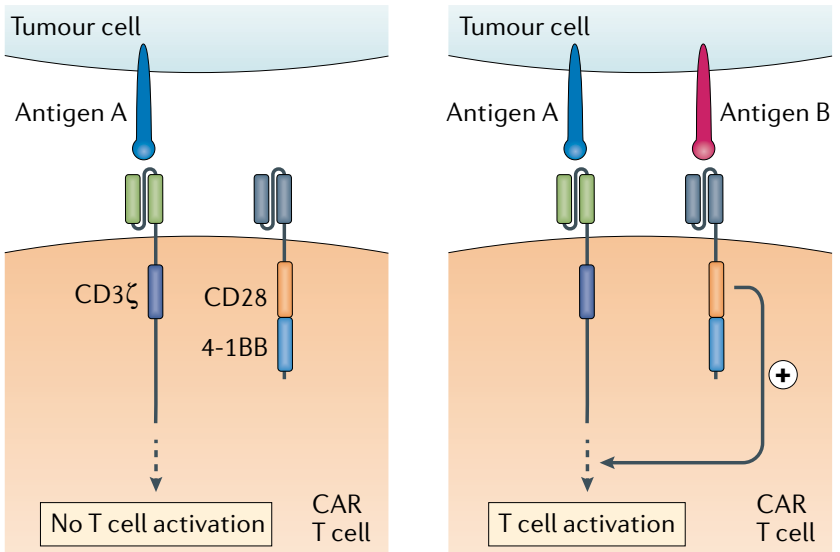

Split CARs: one receptor with a $\mathrm{CD} 3 \zeta$ activation domain and a second with a co-stimulatory domain, requiring binding to multiple TAAs for CAR T cell activation c

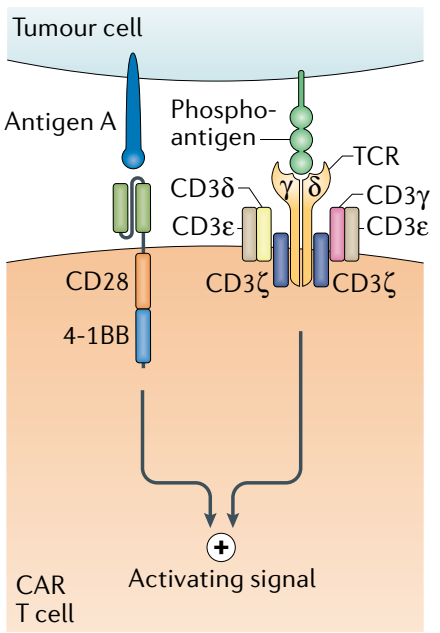

Anti-phosphoantigen $\delta \gamma T$ cells with chimeric co-stimulatory receptor targeting a TAA

\section{d}

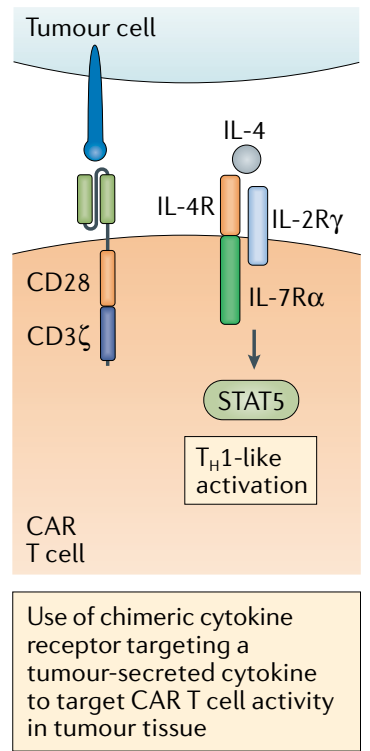

Fig. 3 | Overcoming on-target, off-tumour toxicities of CAR T cells. Chimeric antigen receptor (CAR) T cells are typically designed to target tumour-associated antigens (TAAs); however, expression of these antigens on healthy tissues can result in 'on-target, off-tumour' CAR T cell-mediated toxicities. A | Engineering strategies aiming to overcome this include mechanisms whereby the tumour specificity of CAR T cells is enhanced by ensuring dependency of functional activation on the recognition of multiple TAAs (a), the absence of an antigen selectively expressed on non-malignant cells (b), or the presence of factors that are typically enriched on tumour cells, such as the phosphoantigens that can be recognized via $\gamma \delta \mathrm{T}$ cell receptors (TCRs) (c), or in the tumour microenvironment (TME) such as the immunosuppressive cytokine IL-4 (d). B |Alternative strategies are based on b

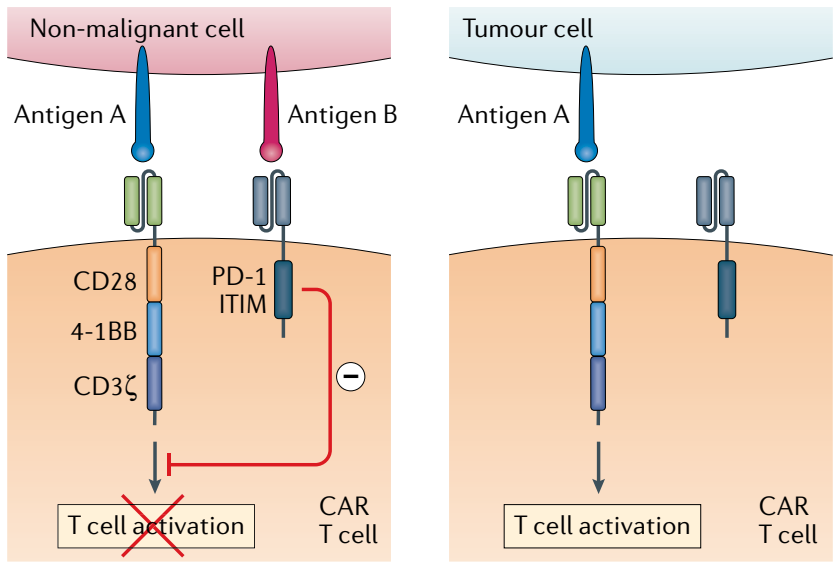

iCARs: inclusion of inhibitory CAR against a specific non-tumour antigen, requiring the absence of this antigen on tumour cells for CAR T cell activation

\section{B Logic gating and/or conditional expression systems}

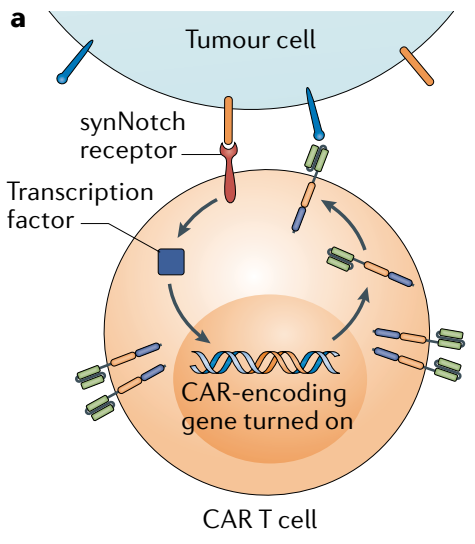

SynNotch CARs: in which a receptor for one TAA drives expression of a second receptor for a different TAA, requiring binding to multiple TAAs for CAR T cell activation

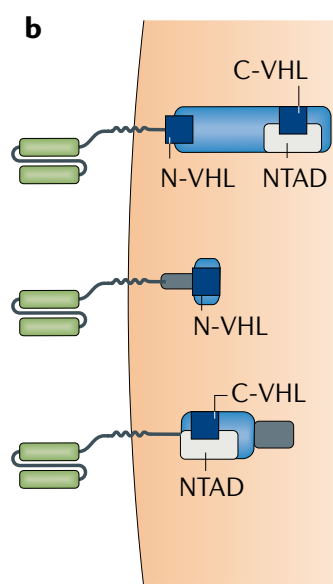

HIF-CARs designed to be expressed selectively in the hypoxic TME logic gating and/or conditional expression systems, whereby expression of a CAR targeting a particular TAA is dependent on activation of another engineered transgenic receptor, such as synthetic Notch (synNotch) receptors, by a different TAA (a) or is driven by a factor associated with the TME such as hypoxia (b). C-VHL, HIF1a C-terminal von Hippel-Lindau tumour suppressor protein recognition site; HIF; hypoxia-inducible factor; iCAR, inhibitory chimeric antigen receptor; IL-2R $\gamma$, IL-2 receptor $\gamma$ chain; IL-4R, IL-4 receptor; IL-7Ra, IL-7 receptor a chain; ITIM, immunoreceptor tyrosine-based inhibitory motif; NTAD, HIF1 a N-terminal transactivation domain; N-VHL, HIF1a N-terminal von Hippel-Lindau tumour suppressor protein recognition site; PD-1, programmed cell death 1; STAT5, signal transducer and activator of transcription $5 ; \mathrm{T}_{H} 1$, T helper 1 . 
favourable antitumour and safety profiles. For example, $\gamma \delta \mathrm{T}$ cells are a T cell subset that have an innate tumoursensing capacity by virtue of expression of TCRs with $\gamma \delta$ subunits (rather than the more common $\alpha \beta$ subunits), which recognize phosphoantigens that are characteristic of tumour cells with dysregulated metabolism ${ }^{105}$ (FIG. 3Ac). Transduction of $\gamma \delta$ T cells with a GD2-targeted CAR harbouring only a co-stimulatory domain resulted in cytolytic responses against $\mathrm{GD} 2^{+}$neuroblastoma cells but not $\mathrm{GD} 2{ }^{+}$non-malignant tissues in vitro (because the $\mathrm{CD} 3 \zeta$ signal was only provided by tumour cells that engaged the endogenous $\gamma \delta \mathrm{TCR})$, illustrating the potentially improved safety profile of this approach ${ }^{106}$.

One particularly creative and ambitious approach to reducing on-target, off-tumour toxicities is to genetically modify the non-malignant tissues to remove the target antigen. CAR T cell treatment for AML is limited by the lack of known cell-surface protein antigens expressed on the cancer cells that are not also present on essential neutrophils and/or essential haematopoietic progenitor cells. Kim et al. ${ }^{107}$ circumvented this barrier by using the clustered regularly interspaced short palindromic repeats (CRISPR) and CRISPR-associated protein 9 (Cas9) (CRISPR-Cas9) genome-editing system to knockout the seemingly non-essential CD33 surface marker in non-malignant haematopoietic progenitor cells. When anti-CD33 CAR T cells were subsequently administered to mice with $\mathrm{CD} 33^{+}$AML, the modified CD33non-malignant bone marrow and myeloid cells were not targeted. The investigators of this study noted that they are planning a clinical trial designed to investigate this approach ${ }^{107}$.

\section{Improving the efficacy of CAR T cells Haematological malignancies}

The most pronounced clinical responses obtained with CAR $T$ cells to date have been observed in patients with certain haematological malignancies, predominantly $\mathrm{CD} 19^{+}$large B cell lymphoma or ALL, although antigen escape and limited persistence of CAR T cells in vivo can restrict the durability of responses and thus the efficacy of CAR T cell therapy in these diseases. Many engineering strategies are being used to address these barriers and to expand the use of CAR T cell therapy in patients with $\mathrm{CD} 19^{-}$haematological malignancies.

Countering antigen escape. Despite high rates of initial response, antigen escape - that is, complete or partial loss of target antigen expression by the cancer cells is observed in a notable proportion of patients treated with CAR T cells. For example, reports from multiple trials and institutional studies have indicated that $7-25 \%$ of patients treated with CD19-targeted CAR T cells relapsed with $\mathrm{CD}^{-} 9^{-}$disease ${ }^{108}$. Mechanisms of CD19 loss include mutations and splice variants of the CD19 gene as well as switching of tumour cells from lymphoid (B cell) lineage to a CD19- myeloid one ${ }^{109}$. Researchers are currently attempting to address antigen escape with a range of combinatorial strategies targeting multiple antigens (FIG. 4Aa-c).

Sequential treatment of patients with different CAR $\mathrm{T}$ cell products targeting alternative antigens have been clinically useful ${ }^{110}$, although engineering a single CAR T cell product that has specificity for multiple targets is an attractive strategy. Multi-target CAR T cell therapies can be created by mixing different CAR $\mathrm{T}$ cell products targeting single antigens prior to infusion or by transducing T cells with multiple CAR constructs ${ }^{9}$. Alternatively, bi-specific CAR T cells can be engineered by designing a single CAR molecule with two (or more) distinct binding domains ${ }^{9}$ and CD19/CD20 or CD19/CD22 bi-specific CAR T cells have demonstrated clinical efficacy in patients with B cell malignancies ${ }^{111,112}$ (FIG. 4Aa). A number of clinical trials designed to test strategies to avoid or delay CAR T cell-associated CD19 antigen escape, including via co-targeting of both CD19 and CD20 (NCT03398967 and NCT03019055) or CD19 and CD22 (NCT03614858, NCT03593109, NCT03468153, NCT03448393, NCT03398967, NCT03330691, NCT03289455, NCT03287817, NCT03241940 and NCT03233854), are currently ongoing worldwide.

Another multi-targeted strategy involves further modification of CAR T cells to secrete bi-specific T cell engagers (BiTEs) (FIG. 4Ab). BiTEs typically consist of two scFvs, one specific to CD3 and the other to a TAA, connected by a flexible linker; therefore, these agents can physically link a T cell to a cancer cell. Notably, the CD19targeted BiTE blinatumomab is currently approved by the FDA for the treatment of ALL ${ }^{113}$. Researchers have demonstrated BiTE-secreting CAR T cells to be effective in overcoming heterogeneity in antigen expression and circumventing antigen escape in preclinical models of leukaemia and solid tumours ${ }^{94,114}$.

Creating CAR T cells that can elicit an endogenous immune response is an alternative approach to generating $\mathrm{T}$ cell responses against multiple TAAs. These agents are referred to as armoured CAR T cells and are co-modified with immunomodulatory agents that engage and modulate other cells of the host's immune system. CAR T cells modified to express the proinflammatory molecule CD40 ligand (CD40L) provide one example ${ }^{115,116}$ (FIG. 4DC). As well as having enhanced intrinsic functionality owing to CD40L co-stimulation, these cells are capable of activating professional antigen-presenting cells and increasing the immunogenicity of tumour cells via engagement of CD40, thereby promoting the recognition and elimination of tumour cells by endogenous, unmodified $\mathrm{T}$ cells ${ }^{115,116}$. Another example comes from CAR T cells modified to secrete soluble herpesvirus entry mediator (HVEM, also known as TNFRSF14), which binds to the inhibitory receptor B lymphocyte and $\mathrm{T}$ lymphocyte attenuator (BTLA) on B cell lymphoma cells, thereby causing tumour suppression ${ }^{117}$.

Increasing CAR T cell persistence. With regard to prolonging the persistence of CAR T cells in patients, one promising strategy is predicated on the use of T cell populations with higher percentages of less differentiated $\mathrm{T}$ cell subsets that have a greater proliferative capacity, such as naive $\mathrm{T}$ cells, stem cell memory $\mathrm{T}\left(\mathrm{T}_{\mathrm{SCM}}\right)$ cells and central memory T $\left(\mathrm{T}_{\mathrm{CM}}\right)$ cells $^{50,118}$ (FIG. 4Ba). Preclinical studies using CAR $\mathrm{T}$ cells generated from preselected naive $\mathrm{T}$ cell populations or manufactured in the presence of kinase inhibitors to generate CAR T cells with 
a less-differentiated phenotype have revealed the superior engraftment, proliferation and antitumour activity of these cells compared with traditional CAR T cell products $^{50,119,120}$. Furthermore, infusion of CAR T cells into patients at a defined 1:1 $\mathrm{CD}^{+}$to $\mathrm{CD}^{+} \mathrm{T}$ cell ratio has been shown to result in cell dose-related increases in CAR T cell expansion and fewer toxicities ${ }^{121,122}$. Trials designed to test CAR $\mathrm{T}$ cell products selectively generated from $\mathrm{CD}^{+} \mathrm{T}_{\mathrm{CM}}$ cells are currently underway $(\mathrm{NCT} 01087294)^{123}$.

\section{A Overcoming antigen escape or heterogeneity via targeting of multiple antigens}

a

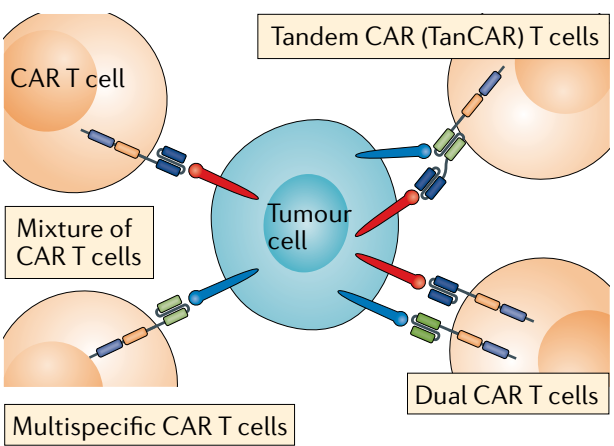

b

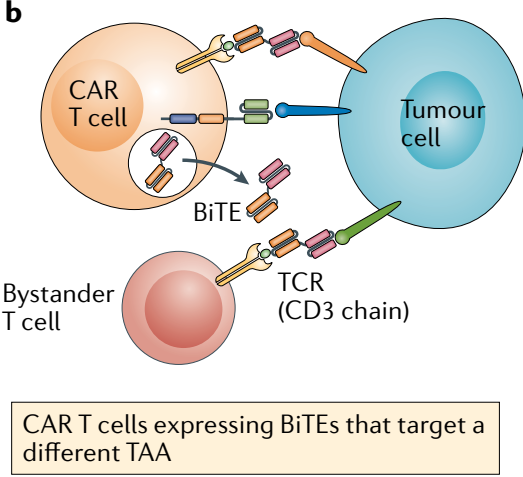

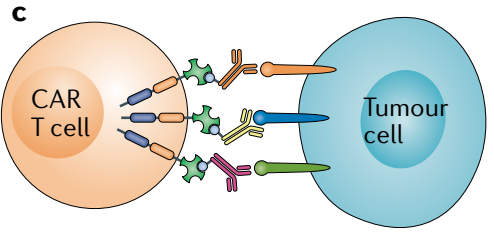

CARs that bind common adapter molecules or immunoglobulin Fc motifs enable simultaneous targeting of multiple antigens with a single CAR construct

\section{B Enhancing in vivo persistence}

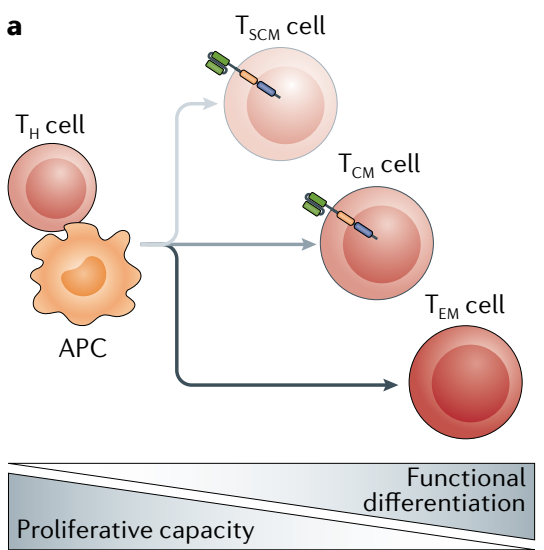

Use of less-differentiated T cell subsets with greater proliferative potential

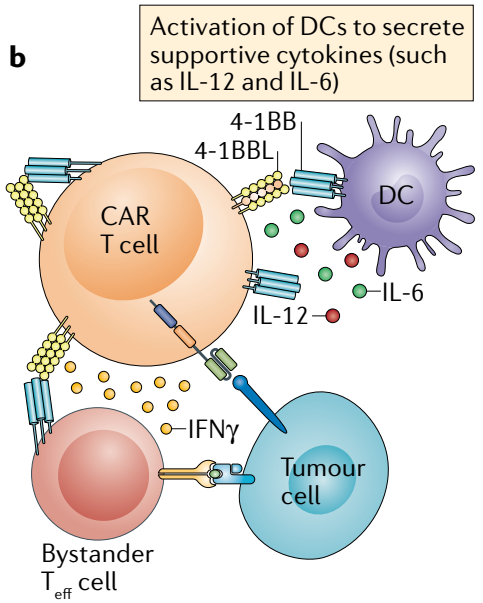

\section{Increasing tumour homing and penetration}

a

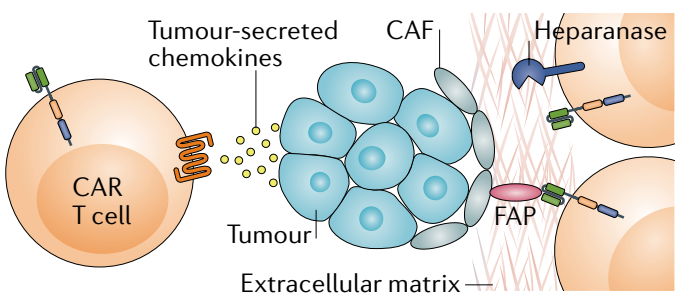

Extra

\begin{tabular}{|l|}
\hline Expression of chemokine \\
receptors, such as \\
CSF-1R, CCR4 or \\
CCR2b, in CAR T cells \\
\hline
\end{tabular}

Targeting stromal barriers with FAP-targeted or heparanase-secreting CAR T cells

D Overcoming immunosuppression in the TME

a

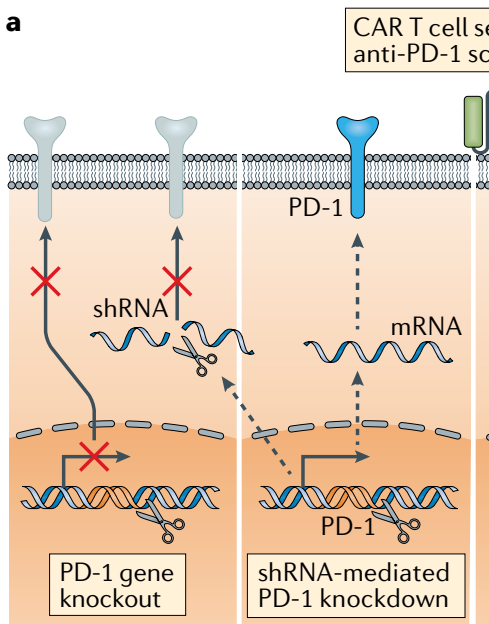

Overcoming inhibitory immune checkpoints such as PD-1 b

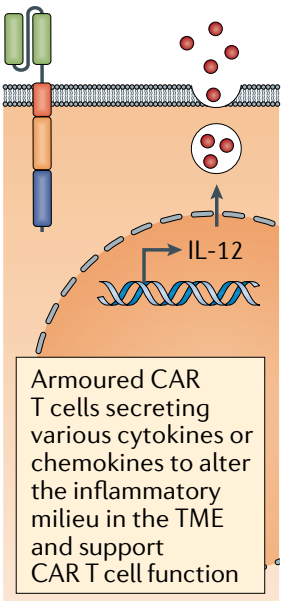

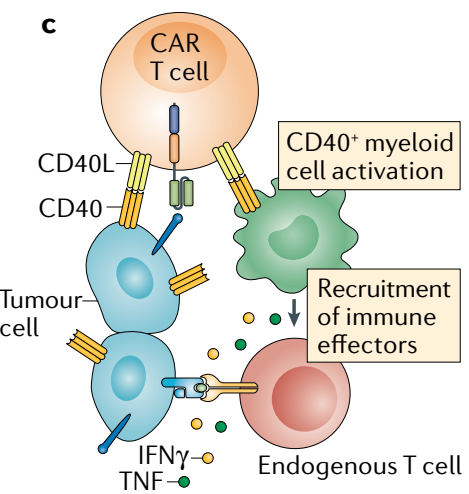

Armoured CAR T cells co-expressing immunomodulatory ligands (such as $\mathrm{CD} 40 \mathrm{~L}$ ) can alter the inflammatory milieu and increase the immunogenicity of tumours 
Co-stimulation is a vital part of effective CAR T cell function, but excess co-stimulatory signalling might decrease the persistence of CAR T cells in patients. Thus, engineering strategies are being developed to address this issue, including altering the co-stimulatory domain of the CAR to decrease the intensity of the signal, for example, by mutating some of the immunoreceptor tyrosine-based activation motifs within the CD28 domain $^{124-126}$. Modulation of the co-stimulatory signal can also be achieved through co-expression of ligands for auto-stimulation and trans-stimulation of CAR T cells. For example, seeking to combine the rapid activatory kinetics afforded by the CD28 co-stimulatory domains and the better persistence associated with the 4-1BB domain, Zhao et al. ${ }^{127}$ demonstrated that co-expression of 4-1BBL on CAR T cells with CD28 co-stimulatory domains results in armoured CAR T cells with a longer duration of persistence, decreased expression of exhaustion markers and a high $\mathrm{CD}^{+}$to $\mathrm{CD} 4^{+} \mathrm{T}$ cell ratio compared with CAR T cells expressing only CD28-based or 4-1BB-based CARs (FIG. 4Bb). These 4-1BBL-expressing CAR T cells are currently being tested in clinical trials in patients with relapsed CD $19^{+}$haematological malignancies (NCT03085173) $)^{128}$.

\section{Solid tumours}

In contrast to the striking successes achieved with CAR $\mathrm{T}$ cells in the treatment of patients with haematological malignancies, no equivalent successes have been demonstrated to date in patients with solid tumours, which collectively account for $\sim 90 \%$ of cancer-related deaths ${ }^{129}$. The disappointing results in patients with solid tumours can be attributed to several factors; the lack of suitable tumour-specific antigens - or TAAs with expression profiles that are likely to be associated with tolerable on-target, off-tumour toxicities - is an obvious barrier to effective CAR T cell therapy for solid tumours. Nevertheless, the collective lack of efficacy observed with diverse CAR T cell products targeting

\footnotetext{
$\varangle$ Fig. 4 | Improving the efficacy of CAR T cell therapy. Several innovative engineering strategies have been used to enhance the efficacy of chimeric antigen receptor (CAR) T cells. A |CAR T cell products designed to target multiple different tumour-associated antigens (TAAs) (a) can overcome antigen escape or heterogeneity; variations on this approach are predicated on the use of CAR T cells engineered to co-express and secrete bi-specific T cell engagers (BiTEs) (b) or the use of CARs targeting adapter molecules that can be linked to a range of soluble antigen-recognition moieties to enable simultaneous recognition of multiple antigens with a single CAR (c). B $\mid$ The in vivo persistence of CAR T cells can be enhanced by using less-differentiated T cell subsets (a) or by engineering CAR T cells to express factors that foster a supportive microenvironment such as 4-1BB ligand (4-1BBL) (b). C | The trafficking and/or penetration of CAR T cells into solid tumours can be improved by engendering these cells with the ability to respond to tumour-associated chemokines (a) or to target physical barriers present in the tumour microenvironment (TME) (b). D | Finally, CAR T cells can be engineered to overcome the immunosuppressive factors present in the TME, for example, by circumventing the activity of inhibitory immune checkpoints, including programmed cell death 1 (PD-1) (a), or by promoting an inflammatory milieu via the expression of cytokines (b) or other immunostimulatory factors, such as CD40 ligand (CD40L) (c). APC, antigen-presenting cell; CAF, cancer-associated fibroblast; CCR2b, CC-chemokine receptor 2b; CCR4, CC-chemokine receptor 4; CSF-1R, macrophage colony-stimulating factor 1 receptor; CSR, chimeric switch receptor; DC, dendritic cell; DNR, dominant negative receptor; FAP, fibroblast activation protein; scFv, single-chain variable fragment; shRNA, short hairpin RNA; $\mathrm{T}_{\mathrm{CM}}$, central memory T cells; TCR, T cell receptor; $\mathrm{T}_{\text {eff }}$ cell, effector T cell; $\mathrm{T}_{\mathrm{EM}}$ cell, effector memory $T$ cells; $T_{H}$ cell, $T$ helper cell; $T_{S C M}$ cell, stem cell-like memory $T$ cell.
}

several different solid tumour antigens suggests the existence of general barriers that could potentially be surmounted with additional CAR T cell engineering. The complicated structure and cellular milieu of solid tumours influences both tumour biology and response to therapy ${ }^{130}$. Solid tumours reside in tissues with lower numbers of endogenous $\mathrm{T}$ cells than lymphoid tissues and perhaps also lower levels of homeostatic cytokines and other T cell-supportive factors normally derived from the bone marrow and lymph node stroma. The structure of the solid tumour stroma can pose a physical barrier to CAR T cell penetration. In addition, suppressive immune cells, such as regulatory $\mathrm{T}\left(\mathrm{T}_{\text {reg }}\right)$ cells and myeloid-derived suppressor cells, and immunosuppressive ligands, such as programmed cell death 1 ligand 1 (PD-L1), present in the TME might all quell intrinsic antitumour immune responses as well as CAR T cell responses.

\section{Overcoming antigen heterogeneity in solid tumours.} Numerous strategies have been developed to overcome the antigen heterogeneity of solid tumours, some of which mirror the aforementioned strategies to overcome antigen escape in haematological malignancies. For example, anti-EGFR BiTEs have been shown to increase the efficacy of anti-EGFRvIII CAR T cells in mouse models of glioblastoma and also of antifolate receptor- $\alpha$ CAR T cells in preclinical models of ovarian, colon or pancreatic cancer ${ }^{99,131}$. Several technologies have been developed to create universal CARs for which adapter elements are used as ligands to enable the targeting of multiple antigens with a single CAR T cell population (FIG. 4Ac). For example, avidin-linked CARs (named biotin-binding immune receptors) in combination with biotinylated antibodies can be used not only to control CAR T cell activity similar to a safety switch but also to target multiple antigens, either sequentially or simultaneously ${ }^{132,133}$. Similar approaches involve the use of CARs with scFvs that recognize a fluorescein isothiocyanate fluorophore conjugated to TAA-binding molecules in order to target multiple antigens simultaneously ${ }^{134-137}$. Likewise, CARs that incorporate $\mathrm{F} c \gamma \mathrm{Rs}$ as the antigen-binding domain enable the use of therapeutic TAA-binding antibodies to target multiple antigens with a single CAR molecule ${ }^{138}$. In the SUPRA (split, universal and programmable) CAR system ${ }^{139}$, leucine zipper motifs are used to match CARs (zipCAR) with free scFvs (zipFv), again enabling simultaneous targeting of multiple antigens as well as the inclusion of multiple antigen logic gates and attenuation of CAR $\mathrm{T}$ cell activation (the CAR T cells are only active when zipFv are present). These and other technologies might provide a means to successfully target heterogeneous solid tumours in patients while minimizing off-tumour toxicities.

Increasing trafficking to solid tumours. Numerous engineering strategies have been developed to improve CAR T cell trafficking to solid tumours. In patients, CAR $\mathrm{T}$ cells have been infused directly to tumours at various anatomical sites, including the brain ${ }^{27}$, breast ${ }^{102}$, pleura $^{140}$ and liver ${ }^{141,142}$, in order to avoid the requirement 
for recruitment from the blood, with some promising responses observed. Local infusion of CAR T cells might also limit on-target, off-tumour toxicities ${ }^{143}$. This general approach might be limited to single tumour lesions or oligometastatic disease and might be more resource intensive than more established local therapies; however, locally delivered CAR T cells have the potential to expand and traffic to other tumour sites and to promote responses of endogenous immune cells against tumours. Indeed, in two patients who received infusions of $\mathrm{T}$ cells transduced with mRNA encoding an anti-mesothelin $\mathrm{CAR}$, one of whom received both intravenous and regional infusions, evidence of an evoked antitumour humoral response involving multiple additional antigens was detected ${ }^{140}$. Similar results have not yet been widely reported in studies involving the intratumoural delivery of CAR T cells, but this result illustrates the potential to use localized delivery to initiate systemic antitumour immune responses. Nevertheless, many metastatic solid tumours are not amenable to localized therapy and thus efforts to engineer CAR T cells with an intrinsic ability to traffic to sites of disease are underway.

Numerous chemokines mediate immune cell trafficking ${ }^{144}$, and modulation of chemokine signalling has been explored to enhance $\mathrm{T}$ cell localization to tumours (FIC. 4 Ca). In a preclinical study ${ }^{145}$, expression of the macrophage colony-stimulating factor 1 receptor (CSF-1R) in CAR T cells made these cells responsive to CSF-1 (a monocyte-recruiting chemokine that is enriched in many solid tumours), which enhanced the proliferative effects of CAR signalling without compromising cytotoxicity and did not induce transdifferentiation to the myeloid lineage. Forced expression of the CC-chemokine receptor 4 (CCR4), which is typically expressed on $\mathrm{T}$ helper cells and $\mathrm{T}_{\text {reg }}$ cells and is activated by CC- chemokine ligand 17 (CCL17) and CCL22 (which are secreted by Reed-Sternberg cells of Hodgkin lymphoma), enhanced both CAR T cell migration to tumours and antitumour activity in a mouse xenograft model of Hodgkin lymphoma ${ }^{146}$. Similarly, forced expression of CCR2b, which is the receptor for CCL2 (a chemokine overexpressed in multiple types of solid tumours), increased the infiltration of anti-GD2 CAR $\mathrm{T}$ cells into neuroblastoma xenograft tumours by more than tenfold ${ }^{147}$ and anti-mesothelin CAR T cell infiltration into mesothelioma xenografts by more than 12-fold, with associated increases in antitumour activity ${ }^{148}$.

Overcoming physical barriers in the solid tumour microenvironment. Several approaches have been explored to enhance the ability of CAR T cells to penetrate physical barriers to enter into the TME of solid cancers and negotiate inhibitory stromal structures (FIG. $4 \mathrm{Cb}$ ). The protease fibroblast activation protein (FAP) is expressed by many tumour-associated stromal fibroblasts and has a role in remodelling of the tumour extracellular matrix (ECM), making this prolyl endopeptidase an attractive target for increasing immune-cell infiltration into tumours. Efforts to target FAP-expressing stromal cells with CAR T cells have had mixed results. In one study, FAP-targeted CAR $T$ cells caused cachexia and bone toxicity via effects on $\mathrm{FAP}^{+}$stromal cells in the bone marrow and had limited effects on the progression of various tumour types in immunocompetent mice ${ }^{149}$. However, in another study using immunocompetent mouse models, FAP-targeted CAR T cells with a different anti-FAP scFv decreased tumour growth when administered in combination with a vaccine, without severe toxicities ${ }^{150}$. Thus, the efficacy and toxicity profile of FAP-targeted CAR T cells requires further investigation.

Engineering CAR T cells to secrete ECM-modifying enzymes is another approach to facilitating the penetration of these cells into solid tumours. Anti-GD2 CAR $\mathrm{T}$ cells engineered to degrade heparin sulfate proteoglycans in the ECM through expression of heparinase had an improved capacity to infiltrate xenograft tumours in mice and prolonged survival as compared with CAR T cells lacking heparinase expression ${ }^{151}$. Other ECM-degrading enzymes are also under exploration for a role in CAR T cell therapy ${ }^{152}$. Caution is warranted, however, following the observation that addition of the pegylated form of the ECM-degrading enzyme hyaluronidase (PEGPH20) to chemotherapy decreased the overall survival of patients with pancreatic adenocarcinoma in one trial ${ }^{153}$, although this effect was not seen in a second trial using a different chemotherapy regimen ${ }^{154}$. Of note, patients who received PEGPH20 in these trials required low molecular weight heparin supplementation to compensate for an increased risk of thromboembolic events, presenting the possibility that $\mathrm{T}$ cells engineered to modify the ECM might also increase the risk of such events. Thus, while ECM modification is an exciting frontier in CAR T cell therapy for solid tumours, caution is warranted given the complicated and currently unpredictable effects of ECM-modifying enzymes.

Overcoming $T$ cell inhibitory signals. In addition to localizing to the tumour, CAR T cells need to overcome direct T cell inhibitory signals present in the TME. While multiple inhibitory signals can be present in the TME, the best characterized pathway involves PD-1. PD-1 is an immune-checkpoint receptor expressed on activated $\mathrm{T}$ cells and, when bound by PD-L1, which can be expressed by tumour cells as well as other cell types, induces T cells to adopt an exhausted, ineffective phenotype. Inhibition of the PD-1 pathway can result in dramatic clinical benefit in patients with certain types of cancer $^{155}$. Notably, expression of PD-1 and other inhibitory receptors has been demonstrated to be a mechanism of CAR T cell dysfunction ${ }^{156}$, and numerous groups have demonstrated increased efficacy of CAR T cell therapy with coadministration of antibodies that inhibit the PD-1 pathway in preclinical models $\mathrm{s}^{157}$ and in subsets of patients with $\mathrm{ALL}^{158}$ or diffuse large B cell lymphoma ${ }^{159}$. Similarly, dramatic antitumour responses have been observed in a subset of patients who received anti-PD-1 antibodies following mesothelin-targeted CAR T cell therapy ${ }^{160}$.

Rather than use CAR T cells in combination with established immune-checkpoint inhibitors, researchers have demonstrated successful strategies to genetically engineer disruption of the PD-1 pathway into CAR T cells themselves (FIG. 4Da). For example, antimesothelin CAR T cells transduced with a truncated, 
dominant-negative $\mathrm{PD}-1$ receptor lacking intracellular signalling domains, which is able to bind to PD-L1 but is incapable of conveying inhibitory signals, resist PD-L1induced exhaustion and prolong survival in mice bearing xenograft pleural mesotheliomas as compared with CAR T cells lacking this decoy receptor ${ }^{161}$. An alternative approach involves $\mathrm{PD}-1$ switch receptors, in which the extracellular domain of PD-1 is fused to the transmembrane and intracellular signalling domain of CD28. Accordingly, these switch receptors transduce an activating co-stimulatory signal when stimulated by $\mathrm{PD}-\mathrm{L} 1$ as well as acting as dominant-negative inhibitors of immunosuppressive signalling via endogenous PD-1 molecules in T cells ${ }^{162-164}$ or CAR T cells ${ }^{165}$ used for adoptive cell therapy, thereby enhancing efficacy in various preclinical models. CAR T cells have also been engineered to secrete antagonistic IgG1 antibodies that can bind to PD-L1 on tumour cells and antigenpresenting cells, which prevents $\mathrm{T}$ cell exhaustion and recruits NK cells that can mediate antibody-dependent cellular cytotoxicity to tumours, as demonstrated in a mouse xenograft model of renal cell carcinoma ${ }^{166}$. Similarly, CAR T cells engineered to secrete antagonistic anti-PD-1 scFvs have enhanced functionality and prolong survival in xenograft $\mathrm{t}^{167,168}$ and immunocompetent syngeneic mouse models of PD-L1 ${ }^{+}$haematological or solid cancers ${ }^{167}$. Notably, these scFvs can also bind to and prevent suppression of endogenous bystander $\mathrm{T}$ cells in the $\mathrm{TME}^{167}$, demonstrating the potential for combining CAR $\mathrm{T}$ cell technologies with immunecheckpoint inhibition as a means of both enhancing CAR $T$ cell function and enlisting endogenous antitumour $\mathrm{T}$ cell populations.

In addition to physical antagonism of the PD-1PD-L1 pathway, gene silencing techniques involving short hairpin RNAs ${ }^{161}$ or CRISPR-Cas9 (REF. ${ }^{169}$ ) have been used to delete the gene encoding PD-1 (PD1) in CAR T cells. Notably, however, a potential role of PD-1 in $\mathrm{T}$ cell activation has been reported ${ }^{170,171}$, warranting further investigation of the effects of these approaches on CAR T cell function. Similarly, short hairpin RNAmediated downregulation of the inhibitory receptor CTLA-4 improved the function of first-generation, $\mathrm{CD} 3 \zeta$-signalling CAR $\mathrm{T}$ cells but had no functional effect on second-generation, CD28-CD3 $\zeta$-signalling CAR T cells ${ }^{172}$.

CAR T cells have also been engineered to counter the actions of adenosine in the TME, which can activate adenosine receptor $\mathrm{A}_{2 \mathrm{~A}}$ and thereby inhibit $\mathrm{T}$ cell function, through expression of a small-peptide disrupter of the interaction between the $\mathrm{A}_{2 \mathrm{~A}}$-activated kinases PKA and ezrin ${ }^{173}$, siRNA silencing of $A_{2 A}\left(R_{E F}{ }^{174}\right)$, or with nanoparticles to deliver a small-molecule $\mathrm{A}_{2 \mathrm{~A}}$ antagonist ${ }^{175}$. Furthermore, dominant-negative receptors that act as a sink for immunosuppressive cytokines, such as TGF $\beta$, and thereby enhance CAR T cell function in the TME have been developed ${ }^{176}$. CAR T cells have also been modified to express decoy or switch cytokine receptors that functionally convert inhibitory signals present in the TME into proinflammatory signals. Examples include fusion of IL-4 receptor ectodomains with endodomains of the IL-7 receptor ${ }^{99,177}$ or with the shared $\beta$-subunit of the IL-2 and IL-15 receptors ${ }^{178}$, thus translating inhibitory IL-4 signals into homeostatic IL-7, IL-2 or IL-15 signals (FIG. 3Ad). T cells and CAR T cells engineered to express a dominant-negative version of the proapoptotic receptor Fas have increased resistance to apoptotic signals from the Fas ligand (FasL) present in the TME, resulting in improved antitumour activity in syngeneic mouse models of various solid or haematological cancers ${ }^{179}$.

In addition to inhibitory signals, the availability of oxygen, amino acids (such as tryptophan, arginine and cysteine) and other nutrients influences the metabolism, function and differentiation of T cells, and the general scarcity of these nutrients in the TME can trigger T cell inhibitory pathways or otherwise suppress T cell function $^{180}$. For example, arginine has important roles in T cell function ${ }^{181}$, and competition with cancer cells and other cells of the TME for this amino acid might result in suppression of the antitumour activity of T cells. Accordingly, pre-treatment of TCR-transgenic T cells with arginine in vitro increased the antitumour activity of these cells after infusion into mice with melanomas expressing the cognate antigen ${ }^{181}$. Intriguingly, elevated potassium levels in the TME have been shown to influence nutrient uptake by $\mathrm{T}$ cells, resulting in metabolic shifts and epigenetic changes that preserve $\mathrm{T}$ cell stemness and inhibit the differentiation of these cells towards effector phenotypes ${ }^{182}$. Correspondingly, in vitro pre-treatment with potassium increases the persistence and antitumour activity of adoptively transferred human $\mathrm{T}$ cells in mouse xenograft models ${ }^{182}$. In another example of modification of $\mathrm{T}$ cell metabolism to overcome aspects of the metabolically hostile TME, expression of the antioxidant enzyme catalase enables T cells to overcome granulocyte-mediated oxidative stress in vitro ${ }^{183}$. As these examples illustrate, modifying and engineering $\mathrm{T}$ cell metabolic pathways might provide new opportunities to increase the efficacy of CAR T cells in patients with solid tumours.

Altering the milieu of the solid tumour microenvironment. As an alternative to overcoming inhibitory signals in the TME, CAR T cells can be engineered to provide immunostimulatory signals that enhance the activity of CAR T cells themselves and, in some cases, also induce an endogenous antitumour response through remodelling of the microenvironment of solid tumours. Regardless of the number of TAAs targeted, antigen escape is always a concern with CAR T cell therapy and thus recruitment of endogenous immune cells might be necessary to propagate the antitumour immune response. Augmenting CAR T cells to secrete stimulatory cytokines that not only foster the proliferation, survival and antitumour activity of $\mathrm{T}$ cells but also alter the immune milieu of solid tumours is an area of active investigation. Indeed, numerous cytokines have been explored to create such forms of armoured CAR T cells (FIG. 4Db), which are also known as T cells redirected for universal cytokine killing (TRUCKs) ${ }^{184}$.

In a mouse model of thymoma, preconditioning with cyclophosphamide to facilitate engraftment of antiCD19 CAR T cells resulted in elevated levels of the proinflammatory cytokine IL-12 (REF. ${ }^{185}$ ). IL-12 has a 
multifaceted role in the immune response, including activation of both T cells and NK cells. Subsequently, genetic modification of CAR T cells to express IL-12 resulted in increased cytotoxicity and resistance to $\mathrm{T}_{\text {reg }}$ cellmediated inhibition in vitro, better engraftment without preconditioning chemotherapy and enhanced antitumour activity in vivo ${ }^{185}$. IL-12 expression also resulted in dramatic CAR T cell expansion from limited numbers of cells isolated from umbilical cord blood samples, introducing the possibility of generating CAR T cells from small volumes of cord blood for patients with haematological malignancies undergoing allogeneic haematopoietic stem cell transplantation (HSCT) ${ }^{115}$. In a subsequent study ${ }^{186}$, IL-12-secreting CAR T cells targeting MUC16 $6^{\text {ecto }}$, which is the membrane-retained portion of the serum cancer biomarker mucin-16 (CA-125), were subsequently shown to have increased antitumour activity in xenograft or immunocompetent syngeneic mouse models of ovarian cancer ${ }^{187}$. In these models, the IL-12-secreting armoured CAR T cells had an increased proliferative capacity, better survival and greater cytotoxicity than the parental, unarmoured CAR T cells and were also more resistant to apoptosis and PD-L1-induced dysfunction ${ }^{187}$. Together, these data supported the initiation of a clinical trial

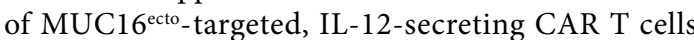
in patients with ovarian cancer (NCT02498912) ${ }^{188}$. In mice xenografted with carcinoembryonic antigen (CEA)-positive and $\mathrm{CEA}^{-}$colon cancer cell lines, IL-12secreting, CEA-targeting CAR T cells have greater anticancer activity than their counterparts lacking IL-12 secretion through promotion of macrophage-mediated killing of the antigen-negative tumour cells ${ }^{189}$. Similarly, IL-12-secreting CARs targeting VEGFR2 have increased efficacy in multiple mouse models of well-vascularized solid cancers ${ }^{190}$. In these models, CAR T cells also altered the immunosuppressive TME by targeting VEGFR2-expressing myeloid-derived suppressor cells; however, the overall antitumour effect was dependent on the expression of the IL-12 receptor on host cells other than T cells and B cells ${ }^{190}$. Notably, lethal toxicity has been observed in clinical trials with systemic IL-12 infusions ${ }^{191}$, and life-threatening haemodynamic instability was observed in a clinical trial of IL-12-secreting tumour-infiltrating lymphocytes ${ }^{192}$; however, expression of IL-12 by CAR T cells via different gene-expression cassettes, for example, from internal ribosome entry sites or promoters with inducible nuclear factor of activated T cells (NFAT) binding motifs, can result in lower levels of IL-12 production ${ }^{193,194}$. Thus, preclinical results have demonstrated the therapeutic promise of IL-12-secreting CAR T cells, although drastic dose-dependent variability in immune responses and toxicities are concerns that must be considered in ongoing and future clinical trials.

IL-15 is required for the differentiation, homeostasis and survival of $\mathrm{T}$ cells and NK cells ${ }^{195}$. In comparison with unarmoured anti-CD19 CAR T cells, the expansion and antitumour activity of armoured CAR T cells expressing IL-15 were increased, with decreased apoptosis and PD-1 expression, in mouse xenograft models of Burkitt lymphoma and in patient-derived B cell chronic lymphocytic leukemia (CLL) samples in vitro ${ }^{195}$. Secretion of IL-15 by anti-IL-13Ra 2 CAR $\mathrm{T}$ cells and by anti-GD2 CAR T cells has subsequently been shown to increase the survival of mice bearing orthotopic glioma xenografts ${ }^{196}$ and metastatic neuroblastoma xenografts ${ }^{197}$, respectively. In a mouse xenograft model of leukaemia, a modified form of IL-15 tethered to the cell membrane of CAR T cells enhanced the anticancer activity and persistence of these cells, engendering them with a memory-like phenotype, and prevented disease relapse ${ }^{198}$. Moreover, fusion of the IL-15 receptor signalling domain to the CD28 co-stimulatory signalling domain of an anti-CD19 CAR resulted in the generation of CAR T cells with enhanced in vivo persistence in preclinical models and induced complete remission in one patient with ALL ${ }^{199}$. Thus, IL-15 can enhance the persistence and activity of CAR $\mathrm{T}$ cells in vivo, although limited evidence of the effects on the TME in immunocompetent models is available for this cytokine. Additionally, concerning exogenous cytokine-independent in vitro clonal expansion has been observed following transduction of mature human lymphocytes to express IL-15, suggesting a potentially dangerous autocrine loop ${ }^{200}$. Moreover, mice with nearubiquitous overexpression of IL-15 develop leukaemia with a T cell or NK cell phenotype ${ }^{201}$. Clinically, toxicities, such as hypotension, thrombocytopenia and elevations in serum aspartate transaminase and/or alanine transaminase levels, have been observed with systemic administration of IL-15 (REF. ${ }^{202}$ ). Given that the half-life of the recombinant IL- 15 used in this trial was $<3$ hours, IL-15 produced continuously by CAR T cells might have different pharmacokinetic and toxicity profiles. Nevertheless, engineering strategies using nanogels to 'backpack' large quantities of IL-15 onto CAR T cells have revealed that high doses of IL-15 can be delivered to tumour sites without systemic toxicities, at least in preclinical models ${ }^{203}$.

IL-18 is a multifunctional cytokine secreted by macrophages. This cytokine can alter the phenotype of T cells and NK cells and has been shown to enhance the engraftment of human effector T cells and inhibit that of immunosuppressive human $\mathrm{T}_{\text {reg }}$ cells in mice ${ }^{204}$. Multiple groups have demonstrated that armoured CAR T cells secreting IL-18 have enhanced functionality compared with their unarmoured counterparts and can alter the inflammatory TME and recruit endogenous immune cells to the tumour in syngeneic immunocompetent mouse models ${ }^{205-207}$. Importantly, IL-18-secreting CAR T cells have been associated with an increased abundance of proinflammatory M1-polarized macrophages in the TME, depletion of anti-inflammatory M2-polarized macrophages and $\mathrm{T}_{\text {reg }}$ cells ${ }^{206}$, and recruitment of endogenous $\mathrm{T}$ cells ${ }^{205}$ in these models. In patients with cancer, systemic administration of IL-18 is well tolerated, with mostly low-grade toxicities ${ }^{208}$. Nevertheless, IL-18 has pathogenic roles in autoimmune diseases ${ }^{209}$ and might also promote tumour progression, angiogenesis, immune escape and metastasis ${ }^{210}$; thus, caution is warranted when investigating IL-18-secreting CAR T cells in the clinic.

Other cytokines have been explored in preclinical models to enhance CAR T cell function. IL-7 and the 
chemokine CCL19 support T cell recruitment and persistence in lymph nodes; engineering of CAR T cells to simultaneously express these proteins, in an attempt to mimic the cytokine environment of lymphoid organs, resulted in increased tumour penetration and antitumour activity of these cells in mice with solid tumours (an effect that was not seen with the expression of either cytokine alone) as well as in increased recruitment of endogenous immune cells and epitope spreading $^{211}$. In other preclinical models, delivery of IL-33 to the TME via CAR T cells enhanced tumour immunogenicity ${ }^{212}$, while secretion of IL-36 $\gamma$ by CAR T cells greatly enhanced CAR T cell expansion and persistence in vivo ${ }^{213}$.

\section{Addressing manufacturing challenges}

The current FDA-approved CAR T cell products as well as the majority of those used in clinical trials to date are manufactured using autologous $\mathrm{T}$ cells obtained from the intended recipient patient. This personalized approach to anticancer therapy has been associated with remarkable successes in the clinic, although generating CAR T cells in this way limits the number of patients who can benefit from this therapy, for several reasons. First, harvesting sufficient numbers of $\mathrm{T}$ cells from patients with cancer can be difficult, as many patients are lymphopenic as a sequalae of the disease or previous chemotherapy. In paediatric patients, chemotherapy also decreases the percentage of naive $T$ cells in peripheral blood, which are important for the clinical activity of CAR T cells ${ }^{214}$, and low levels of this T cell subset can lead to production failure ${ }^{215}$. Second, autologous CAR $\mathrm{T}$ cell products have a lengthy and individualized manufacturing process that might not be feasible for patients with advanced-stage cancer, in whom the disease might progress during manufacturing ${ }^{4,5}$. Finally, failure of CAR T cell production can also be attributable to the characteristics of the apheresis product ${ }^{216}$. For example, disease-related dysfunctions of $\mathrm{T}$ cells $\mathrm{s}^{217}$, such as those described in patients with CLL $^{218,219}$ or solid tumours ${ }^{220}$, can result in unsuccessful manufacturing or inferior products that lead to poor response rates in patients.

CAR T cells engineered from allogeneic donor T cells present an alternative to autologous CAR T cells and could circumvent the manufacturing issues of inadequate cell numbers, suboptimal T cell states and delays in treatment. In patients with haematological malignancies, HSCT is commonly performed, and CAR T cells produced from the original human leukocyte antigen (HLA)-matched allogeneic haematopoietic stem cell donors have been used. Indeed, the treatment of patients with persistent $B$ cell malignancies following allogeneic HSCT using donor-derived leukocytes expressing CD19directed CARs has led to complete and partial remissions $^{221-223}$. GVHD has developed in a subset of patients in a few of these studies ${ }^{222,223}$, although some of these events might have been attributable to prior treatment, and allogeneic CAR T cell therapy has been associated with a lower rate of GVHD than that expected with traditional donor lymphocyte infusion. The CAR constructs used in these studies had a CD28 co-stimulatory domain, and findings in preclinical models have suggested that CARs with a $4-1 \mathrm{BB}$ co-stimulatory domain are associated with a greater risk of GVHD than those with a CD28 co-stimulatory domain ${ }^{224}$. Partially HLA-matched donor-derived viral antigen-specific $\mathrm{T}$ cells are a second potential source of allogeneic $\mathrm{T}$ cells for transduction with CAR constructs ${ }^{50}$. These CAR T cells lack alloreactive potential because the TCR is virus specific and reportedly have promising activity in patients with relapsed B cell leukaemia after allogeneic HSCT $^{225}$.

As observed in the studies discussed above, the engrafted allogeneic donor $\mathrm{T}$ cells can become reactive to cells of the recipient, resulting in $\mathrm{GVHD}^{222}$. Conversely, the host immune cells can also recognize and eliminate the donor T cells. Expression of CARs in alternative allogeneic donor cell types, such as NK cells (which have intrinsic MHC-unrestricted activity), might avoid the development of GVHD, but has been associated with decreased persistence of the CAR-modified cells in patients ${ }^{226,227}$. Nevertheless, researchers are also exploring a variety of engineering solutions to the challenges posed by $\mathrm{T}$ cell alloreactivity, with the goal of engineering universal, 'off the shelf' allogeneic CAR T cells. These CAR T cells could be manufactured in bulk from healthy donors to be readily available for use in a timely manner, while also addressing other production and $\mathrm{T}$ cell quality issues.

One approach to engineering such universal CAR $\mathrm{T}$ cells involves the use of genome-editing techniques to abolish the expression of $\alpha \beta$ TCR and/or MHC class I (MHC I) complexes in allogeneic donor T cells. Knocking out the expression of either the TCR $\alpha$ or $\beta$ chains prevents donor CAR T cells from recognizing host alloantigens, and thus GVHD, because the a $\beta$ TCR heterodimer is necessary for the assembly and activity of the entire TCR complex. Deletion of the a chain through targeted disruption of the TRAC locus has been the most common GVHD-avoidance strategy to date ${ }^{228}$. The $\beta$ chain is encoded by two TRBC genes (TRBC1 and $T R B C 2$ ) and, therefore, knocking out this subunit is potentially more complicated. Editing out MHC I in donor $\mathrm{T}$ cells conversely prevents recognition of these cells by $\mathrm{T}$ cells of the recipient and thus rejection of the graft.

Zinc finger nucleases (ZFN) are artificial restriction enzymes that can be engineered to cause doublestranded DNA breaks at defined locations within the genome. These breaks are subsequently repaired by the non-homologous end joining or homologous recombination DNA repair pathways, which can be exploited to disrupt, and thereby prevent, the expression of the target gene. CAR T cells have been modified using ZFN to eliminate the expression of the TCR $\alpha$ or $\beta$ chains (and thus functional TCRs) ${ }^{229}$, or the HLA-A ${ }^{230}$ or $\beta 2$ microglobulin $(\beta 2 \mathrm{M})^{231}$ subunits of $\mathrm{MHC}$ I; these CAR $\mathrm{T}$ cells did not cause GVHD in preclinical models. Transcription activator-like (TAL) effector nuclease (TALEN) technologies have also been used to knockout the $\mathrm{TCR}^{232}$, and TALEN-edited anti-CD19 CAR T cells have induced remissions in infants with $B$ cell ALL, with no GVHD observed ${ }^{233}$. Fusion proteins comprising a meganuclease conjugated with a TAL repeat DNA recognition domain (termed megaTAL) have also been 
used to disrupt $\alpha \beta T C R^{228}$. However, these gene-editing methods require the design of specific pairs of nucleases, which has limited the widespread use of these systems. Alternatively, the CRISPR-Cas9 platform has been used to prevent the expression of the TCR $\alpha$ chain ${ }^{234,235}$ or a combination of the TCR $\alpha$ and $\beta$ chains, $\beta 2 \mathrm{M}$ and PD- 1 $\left(\right.$ REFS $\left.^{231,236}\right)$ in CAR T cells. Deletion of $\alpha \beta T C R$ and $\beta 2 M$ has also been successfully achieved using a combination of the CRISPR-Cas9 and TALEN platforms, termed Cas-Clover ${ }^{237}$. Insertion of the CAR gene construct into the TRAC locus has also been achieved using CRISPRCas9 in preclinical models, thereby knocking out the TCR and inserting the CAR in one step ${ }^{238}$; this feat has also been achieved using an engineered I-CreI homing endonuclease and an adeno-associated virus donor template $^{239}$. These approaches have the dual advantage of eliminating the endogenous TCR, thereby reducing the risk of GVHD and thus facilitating the use of allogeneic CAR T cells, whilst simultaneously enhancing CAR $T$ cell function by reducing tonic signalling and ensuring uniform cell-surface levels of CAR expression as well as efficient recycling of internalized CARs back to the cell surface.

Beyond genome-editing techniques, alternative genetic engineering approaches have been used to eliminate TCR expression in CAR T cells, including the protein expression blocker (PEBL) system, whereby a 21-amino acid endoplasmic reticulum retention signal linked to an anti-CD3e scFv is used to prevent TCR expression on the cell surface ${ }^{240}$. PEBL has been demonstrated to avoid GVHD without affecting the antitumour activity of CAR T cells in mouse model ${ }^{240}$.

Importantly, none of these techniques enables TCR knockout in $100 \%$ of CAR T cells ${ }^{241}$, thus necessitating further purification of the $\mathrm{T}$ cell products prior to use in patients; however, whether an acceptable level of contaminating TCR-expressing allogeneic donor CAR $\mathrm{T}$ cells exists that will not result in GVHD is currently unknown. Furthermore, knockout of MHC I molecules might lead to NK cell-mediated killing and might not prevent CAR T cell rejection mediated by MHC II molecules, which are expressed in activated T cells. Worldwide, several trials designed to test gene-edited allogeneic CAR T cells in patients with cancer are currently ongoing ${ }^{9}$ (TABLE 1).

A second approach to engineering universal CAR $\mathrm{T}$ cells is to generate them in vitro from mismatched allogeneic haematopoietic progenitor cells, which will subsequently develop into functional mature CAR $\mathrm{T}$ cells in the host. This approach ensures that the MHC restriction of the infused CAR T cells is determined by the host MHC molecules, thereby reducing the risk of GVHD. Naive T cells have been generated from haematopoietic stem cells present in donor bone marrow or cord blood samples, embryonic stem cells or induced pluripotent stem (iPS) cells through culturing with stromal cells and Notch ligands, commonly the OP9 mouse bone marrow stromal cell line and Delta-like ligand 1 (DLL1) or DLL4 (REF. ${ }^{242}$ ). Preclinical studies with CAR-expressing $\mathrm{T}$ cell precursors have demonstrated in vivo maturation of these T cells, with no GVHD observed $^{243,244}$. Importantly, donor T cells that have been reprogrammed to restore pluripotency and create CAR iPS cells are capable of self-renewal and can be banked ${ }^{245}$. Indeed, a single cell-derived CAR iPS master cell line, FT819, has been created by dedifferentiating peripheral T cells into iPS cells followed by biallelic disruption of the TRAC locus with the CAR gene ${ }^{246,247}$. This strategy has

Table 1 | Open clinical trials of genetically engineered allogeneic CAR T cells

\begin{tabular}{|c|c|c|c|}
\hline Target antigen & Disease & $\begin{array}{l}\text { ClinicalTrial.gov } \\
\text { identifier }\end{array}$ & Sponsor \\
\hline \multirow[t]{7}{*}{ CD19 } & R/R CD19+ leukaemia and lymphoma & NCT03166878 & Chinese PLA General Hospital \\
\hline & R/R B-ALL & NCT02746952 & $\begin{array}{l}\text { Institut de Recherches Internationales } \\
\text { Servier }\end{array}$ \\
\hline & $\mathrm{R} / \mathrm{R}$ large $\mathrm{B}$ cell or follicular lymphoma & NCT03939026 & Allogene Therapeutics \\
\hline & R/R NHL or B-ALL & NCT03666000 & Precision BioSciences \\
\hline & $\mathrm{R} / \mathrm{R} B$ cell malignancies & NCT04035434 & CRISPR Therapeutics \\
\hline & $\begin{array}{l}\mathrm{R} / \mathrm{R} \mathrm{B} \text { cell haematological } \\
\text { malignancies }\end{array}$ & NCT03229876 & Shanghai Bioray Laboratory \\
\hline & $\begin{array}{l}\text { Elderly patients ( } \geq 60 \text { years of age) with } \\
\text { R/R CD } 19^{+} \text {B-ALL }\end{array}$ & NCT02799550 & $\begin{array}{l}\text { The Affiliated Hospital of the Chinese } \\
\text { Academy of Military Medical Sciences }\end{array}$ \\
\hline $\begin{array}{l}\text { CD19 and CD20 } \\
\text { or CD22 }\end{array}$ & R/R leukaemia or lymphoma & NCT03398967 & Chinese PLA General Hospital \\
\hline CD123 & Acute myeloid leukaemia & NCT03190278 & Cellectis S.A. \\
\hline \multirow[t]{2}{*}{ BCMA } & Multiple myeloma & NCT03752541 & Shanghai Bioray Laboratory \\
\hline & Multiple myeloma & NCT04093596 & Allogene Therapeutics \\
\hline NKG2D ligands & $\begin{array}{l}\text { Unresectable metastatic colorectal } \\
\text { cancer }\end{array}$ & NCT03692429 & Celyad \\
\hline Mesothelin & Mesothelin ${ }^{+}$solid tumours & NCT03545815 & Chinese PLA General Hospital \\
\hline
\end{tabular}

B-ALL, B cell acute lymphoblastic leukaemia; BCMA, B cell maturation antigen; CAR, chimeric antigen receptor; NHL, non-Hodgkin lymphoma; NKG2D, natural killer cell receptor D; R/R, relapsed and/or refractory. 


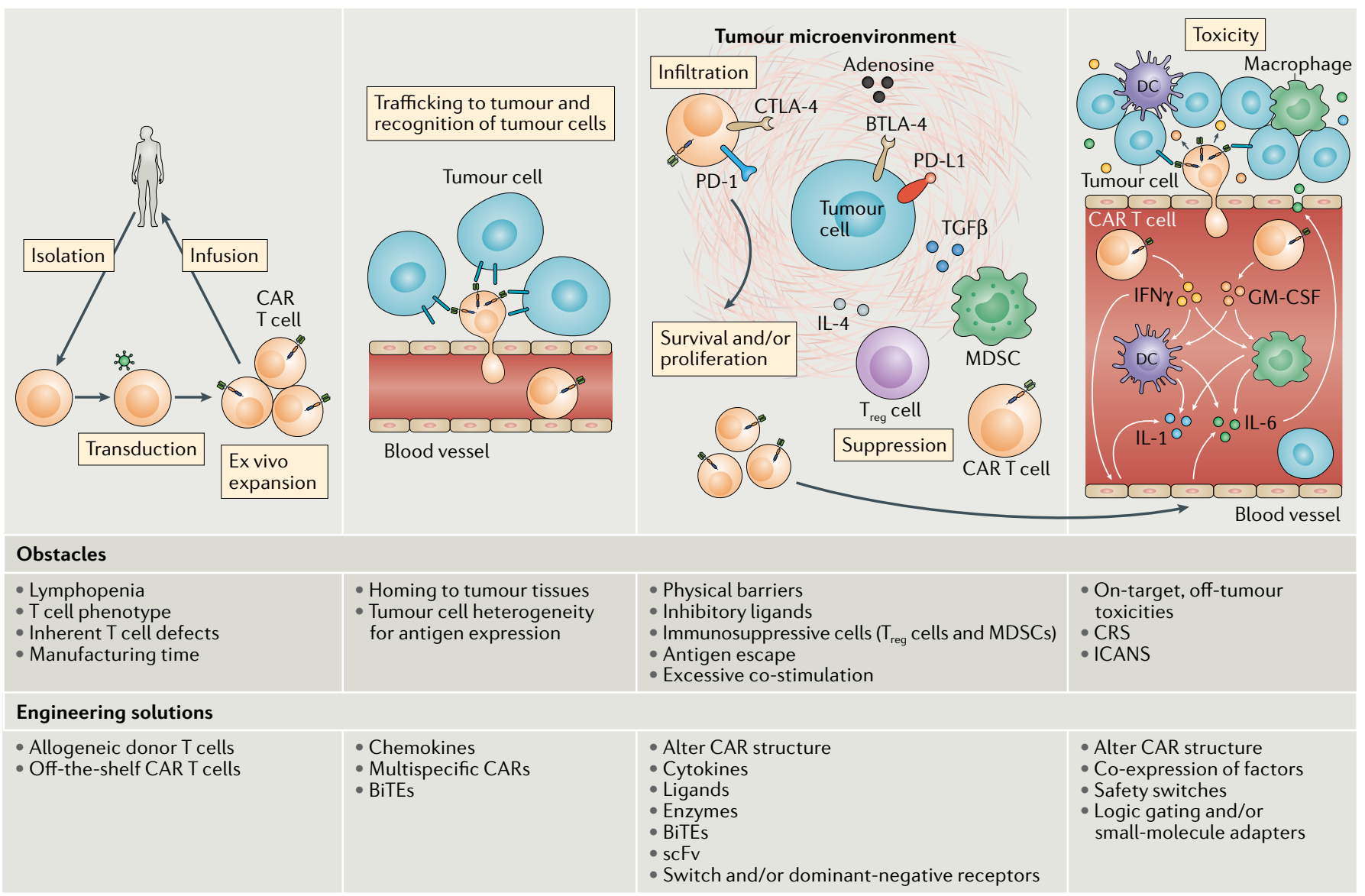

Fig. 5 | Life cycle of a CAR T cell and associated challenges to safe and effective therapy. Many obstacles must be overcome over the life cycle of chimeric antigen receptor (CAR) T cells, including production issues, physical barriers to tumour infiltration, tumour heterogeneity in antigen expression, immunosuppressive factors in the tumour microenvironment and challenging toxicities. Researchers have devised several novel engineering solutions to address each of these issues, as outlined in this figure. BiTEs, bi-specific T cell engagers; BTLA-4, B and T lymphocyte attenuator 4; CRS, cytokine release syndrome; CTLA-4, cytotoxic T lymphocyte protein 4; DC, dendritic cell; GM-CSF, granulocytemacrophage colony-stimulating factor; ICANS, immune effector cell-associated neurotoxicity syndrome; MDSCs, myeloid-derived suppressor cells; PD-1, programmed cell death 1; PD-L1, programmed cell death 1 ligand 1; scFv, single-chain Ig variable fragment; TGF $\beta$, transforming growth factor- $\beta ; \mathrm{T}_{\text {reg, }}$, regulatory $\mathrm{T}$ cells.

resulted in a well-defined, expandable cell product derived from a single source with no native TCR and with expression of the CAR under transcriptional control of the TRAC endogenous regulatory elements.

Eliminating the requirement to generate an autologous CAR T cell product through the aforementioned approaches might avoid treatment delays. In addition, the development of universal CAR T cells will provide opportunities for upscaling of manufacturing and banking of the product. These improvements in manufacturing could potentially expand the access of patients with cancer to CAR $\mathrm{T}$ cell therapies and might also lead to decreases in the substantial costs of CAR T cell therapies.

\section{Future perspectives}

CAR T cells are a new class of therapy and, indeed, a new therapeutic paradigm. The modular design of current CARs (FIG. 1) enables refinements to address the diseasespecific challenges posed by the TME. By using synthetic biology and gene-editing technologies, researchers can efficiently engineer CAR T cells to be safer and more effective (FIG. 5). Moreover, CAR T cells can be used as living $\mathrm{T}$ cell 'micropharmacies' for the targeted delivery of immunomodulatory molecules to the TME.

Importantly, however, increasing the complexity of CAR designs and gene editing of T cells might amplify the risks associated with CAR T cell therapy. For example, viral transduction and the use of gene-editing machineries come with the danger of off-target disruption of genes $^{232}$. Indeed, transformation of $\mathrm{T}$ cells into malignant clones, owing to insertional mutagenesis involving either activation of endogenous proto-oncogenes by viral promoters or disruption of tumour-suppressor genes, is a well-anticipated theoretical risk of any gene therapy. Such transformation through insertional mutagenesis has not been described in patients to date, although viral insertion into genes has been observed clinically in lentivirally transduced CAR T cells used in the treatment of a patient with $\mathrm{CLL}^{248}$. Specifically, the CAR gene was inserted into the TET2 loci and led to a clonal expansion of $\mathrm{T}$ cells ${ }^{248}$; this clonal $\mathrm{T}$ cell population eventually 
contracted spontaneously, but this event highlights the risk of treating patients with genetically modified cells. As experience with adoptive cell transfer accumulates, however, researchers are becoming more comfortable with this risk, and a first-in-human trial has been initiated to evaluate an NY-ESO-1-directed transgenic TCR $\mathrm{T}$ cell product with multiple CRISPR-Cas9 gene edits to remove endogenous TCR and PD-1 (NCT03399448).

Intricate gene editing of cellular products ex vivo also adds complexity to the manufacturing process. Experience with the current CAR T cell manufacturing process has demonstrated that circulating tumour cells harvested together with lymphocytes in the apheresis product can inadvertently be transduced with the CAR construct, which can result in antigen masking via binding of CARs expressed by these tumour cells to TAA on the same cells, resulting in clonal expansion and tumour cell escape in vivo ${ }^{249}$. The CAR T cell technology is the first clinically approved gene therapy, and ongoing monitoring of gene editing-related complications in trials of CAR T cell products will help to further characterize the long-term risks relating to the emerging field of gene editing in medicine and might facilitate the development of solutions to these complications.

Novel engineering approaches might also add to the already high cost of CAR T cell manufacturing. The cost of CAR T cell production, especially the high cost and time associated with the production of clinical grade retroviruses, is only one of many factors that are reflected in the final cost of these therapies; however, methods to reduce manufacturing costs, such as the use of non-viral vectors, might help to improve affordability ${ }^{250}$.

\section{Conclusions}

Exciting approaches are currently under development to increase the efficacy and scope of CAR T cell therapies while improving the safety and facilitating the efficient production of these agents. These promising engineering solutions to optimize CAR T cell biology will lead to the adoption of this technology for more widespread use in anticancer therapy and expand the benefits of CAR $\mathrm{T}$ cells for patients.

Published online 17 December 2019
1. Jackson, H. J., Rafiq, S. \& Brentjens, R. J. Driving CAR T-cells forward. Nat. Rev. Clin. Oncol. 13, 370-383 (2016).

2. Neelapu, S. S. et al. Chimeric antigen receptor T-cell therapy - assessment and management of toxicities. Nat. Rev. Clin. Oncol. 15, 47-62 (2018).

3. Park, J. H. et al. Long-term follow-up of CD 19 CAR therapy in acute lymphoblastic leukemia. N. Engl. J. Med. 378, 449-459 (2018).

4. Maude, S. L. et al. Tisagenlecleucel in children and young adults with B-cell lymphoblastic leukemia. N. Engl. J. Med. 378, 439-448 (2018).

5. Schuster, S. J. et al. Chimeric antigen receptor T cells in refractory B-cell lymphomas. N. Engl. J. Med. 377, 2545-2554 (2017).

6. U.S. Food \& Drug Administration. FDA approves tisagenlecleucel for B-cell ALL and tocilizumab for cytokine release syndrome. fda.gov https://www.fda. gov/drugs/resources-information-approved-drugs/ fda-approves-tisagenlecleucel-b-cell-all-and-tocilizumabcytokine-release-syndrome (2017).

7. U.S. Food \& Drug Administration. FDA approves axicabtagene ciloleucel for large B-cell lymphoma. fda.gov https://www.fda.gov/drugs/resourcesinformation-approved-drugs/fda-approves-axicabtageneciloleucel-large-b-cell-lymphoma (2017).

8. Ramello, M. C., Haura, E. B. \& Abate-Daga, D. CAR-T cells and combination therapies: what's next in the immunotherapy revolution? Pharmacol. Res. 129, 194-203 (2018).

9. Bailey, S. R. \& Maus, M. V. Gene editing for immune cell therapies. Nat. Biotechnol. https://doi.org/ 10.1038/s41587-019-0137-8 (2019)

10. Dwivedi, A., Karulkar, A., Ghosh, S., Rafiq, A. \& Purwar, R. Lymphocytes in cellular therapy: functional regulation of CAR T cells. Front. Immunol. 9, 3180 (2019).

11. Zhang, G. et al. Anti-melanoma activity of T cells redirected with a TCR-like chimeric antigen receptor. Sci. Rep. 4, 3571 (2014).

12. Oren, R. et al. Functional comparison of engineered T cells carrying a native TCR versus TCR-like antibodybased chimeric antigen receptors indicates affinity/ avidity thresholds. J. Immunol. 193, 5733-5743 (2014).

13. Inaguma, Y. et al. Construction and molecular characterization of a T-cell receptor-like antibody and CAR-T cells specific for minor histocompatibility antigen HA-1H. Gene Ther. 21, 575-584 (2014).

14. Rafiq, S. et al. Optimized T-cell receptor-mimic chimeric antigen receptor $\mathrm{T}$ cells directed toward the intracellular Wilms tumor 1 antigen. Leukemia 31, 1788-1797 (2017)

15. Chang, Z. L. et al. Rewiring T-cell responses to soluble factors with chimeric antigen receptors. Nat. Chem. Biol. 14, 317-324 (2018)
16. Xie, Y. J. et al. Nanobody-based CAR T cells that target the tumor microenvironment inhibit the growth of solid tumors in immunocompetent mice. Proc. Natl Acad. Sci. USA 116, 7624-7631 (2019).

17. Smith, E. L. et al. GPRC5D is a target for the immunotherapy of multiple myeloma with rationally designed CAR T cells. Sci. Transl. Med. 11, eaau7746 (2019).

18. Smith, E. L. et al. Development and evaluation of an optimal human single-chain variable fragment-derived BCMA-targeted CAR T cell vector. Mol. Ther. 26, 1447-1456 (2018)

19. Chailyan, A., Marcatili, P. \& Tramontano, A The association of heavy and light chain variable domains in antibodies: implications for antigen specificity. FEBS J. 278, 2858-2866 (2011).

20. Hudecek, M. et al. Receptor affinity and extracellular domain modifications affect tumor recognition by ROR1-specific chimeric antigen receptor T cells. Clin. Cancer Res. 19, 3153-3164 (2013)

21. Lynn, R. C. et al. High-affinity FR $\beta$-specific CAR T cells eradicate AML and normal myeloid lineage without HSC toxicity. Leukemia 30 , 1355-1364 (2016)

22. Liu, X. et al. Affinity-tuned ErbB2 or EGFR chimeric antigen receptor $\mathrm{T}$ cells exhibit an increased therapeutic index against tumors in mice. Cancer Res. 75, 3596-3607 (2015)

23. Caruso, H. G. et al. Tuning sensitivity of CAR to EGFR density limits recognition of normal tissue while maintaining potent antitumor activity. Cancer Res. 75, 3505-3518 (2015)

24. Watanabe, K. et al. Excessively high-affinity singlechain fragment variable region in a chimeric antigen receptor can counteract T-cell proliferation. Blood 124, 4799-4799 (2014)

25. Ghorashian, S et al. Enhanced CAR T cell expansion and prolonged persistence in pediatric patients with ALL treated with a low-affinity CD19 CAR. Nat. Med. 25, 1408-1414 (2019)

26. Ajina, A. \& Maher, J. Strategies to address chimeric antigen receptor tonic signaling. Mol. Cancer Ther. 17, 1795-1815 (2018)

27. Brown, C. E. et al. Regression of glioblastoma after chimeric antigen receptor T-cell therapy. N. Engl. J. Med. 375, 2561-2569 (2016).

28. Lee, L. et al. An APRIL-based chimeric antigen receptor for dual targeting of $\mathrm{BCMA}$ and $\mathrm{TACl}$ in multiple myeloma. Blood 131, 746-758 (2018).

29. Wang, Y. et al. Targeting FLT3 in acute myeloid leukemia using ligand-based chimeric antigen receptor-engineered T cells. J. Hematol. Oncol. 11, 60 (2018)

30. Nakazawa, Y. et al. Anti-proliferative effects of $\mathrm{T}$ cells expressing a ligand-based chimeric antigen receptor against CD 116 on $\mathrm{CD} 34^{+}$cells of juvenile myelomonocytic leukemia. J. Hematol. Oncol. 9 , 27 (2016)

31. Baumeister, S. H. et al. Phase I trial of autologous CAR T cells targeting NKG2D ligands in patients with AML/MDS and multiple myeloma. Cancer Immunol. Res. 7, 100-112 (2019).

32. Hammill, J. A. et al. Designed ankyrin repeat proteins are effective targeting elements for chimeric antigen receptors. J. Immunother. Cancer 3, 55 (2015).

33. Siegler, E., Li, S., Kim, Y. J. \& Wang, P. Designed ankyrin repeat proteins as Her2 targeting domains in chimeric antigen receptor-engineered T cells. Hum. Gene Ther. 28, 726-736 (2017).

34. Han, X. et al. Adnectin-based design of chimeric antigen receptor for $\mathrm{T}$ cell engineering. Mol. Ther. 25, 2466-2476 (2017).

35. Jensen, M. C. \& Riddell, S. R. Designing chimeric antigen receptors to effectively and safely target tumors. Curr. Opin. Immunol. 33, 9-15 (2015).

36. Alabanza, L. et al. Function of novel anti-CD19 chimeric antigen receptors with human variable regions is affected by hinge and transmembrane domains. Mol. Ther. 25, 2452-2465 (2017).

37. James, S. E. et al. Antigen sensitivity of CD22-specific chimeric TCR is modulated by target epitope distance from the cell membrane. J. Immunol. 180, 7028-7038 (2008).

38. Haso, W. et al. Anti-CD22-chimeric antigen receptors targeting B-cell precursor acute lymphoblastic leukemia. Blood 121, 1165-1174 (2013).

39. Qin, L., Zhao, R. \& Li, P. Incorporation of functional elements enhances the antitumor capacity of CAR T cells. Exp. Hematol. Oncol. 6, 28 (2017).

40. Watanabe, N. et al. Fine-tuning the CAR spacer improves T-cell potency. Oncoimmunology 5 e1253656 (2016).

41. Guest, R. D et al. The role of extracellular spacer regions in the optimal design of chimeric immune receptors: evaluation of four different scFvs and antigens. J. Immunother. 28, 203-211 (2005).

42. Hudecek, $M$. et al. The nonsignaling extracellular spacer domain of chimeric antigen receptors is decisive for in vivo antitumor activity. Cancer Immunol. Res. 3 , 125-135 (2015).

43. Hombach, A., Hombach, A. A. \& Abken, $\mathrm{H}$. Adoptive immunotherapy with genetically engineered $T$ cells: modification of the IgG1 Fc 'spacer' domain in the extracellular moiety of chimeric antigen receptors avoids 'off-target' activation and unintended initiation of an innate immune response. Gene Ther. 17 . 1206-1213 (2010).

44. Jonnalagadda, $\mathrm{M}$ et al. Chimeric antigen receptors with mutated IgG4 Fc spacer avoid fc receptor binding and improve T cell persistence and antitumor efficacy. Mol. Ther. 23, 757-768 (2015). 
45. Bridgeman, J. S. et al. The optimal antigen response of chimeric antigen receptors harboring the $\mathrm{CD} 3 \zeta$ transmembrane domain is dependent upon incorporation of the receptor into the endogenous TCR/CD3 complex. J. Immunol. 184, 6938-6949 (2010)

46. Guedan, S. et al. Enhancing CAR T cell persistence through ICOS and 4-1 BB costimulation. JCI Insight 3, 96976 (2018)

47. Dotti, G., Gottschalk, S., Savoldo, B. \& Brenner, M. K. Design and development of therapies using chimeric antigen receptor-expressing T cells. Immunol. Rev. 257, 107-126 (2014).

48. Brocker, T. \& Karjalainen, K. Signals through T cell receptor- $\zeta$ chain alone are insufficient to prime resting T lymphocytes. J. Exp. Med. 181, 1653-1659 (1995).

49. Maher, J., Brentjens, R. J., Gunset, G., Riviere, I. \& Sadelain, M. Human T-lymphocyte cytotoxicity and proliferation directed by a single chimeric TCR /CD28 receptor. Nat. Biotechnol. 20, 70-75 (2002).

50. Sadelain, M., Riviere, I. \& Riddell, S. Therapeutic T cell engineering. Nature 545, 423-431 (2017).

51. Kawalekar, O. U. et al. Distinct signaling of coreceptor regulates specific metabolism pathways and impacts memory development in CAR T cells. Immunity $\mathbf{4 4}$, 712 (2016).

52. Mata, M. et al. Inducible activation of MyD88 and CD40 in CAR T cells results in controllable and potent antitumor activity in preclinical solid tumor models. Cancer Discov. 7, 1306-1319 (2017).

53. Hombach, A. A., Heiders, J., Foppe, M., Chmielewski, M. ¿ Abken, H. OX40 costimulation by a chimeric antigen receptor abrogates CD28 and IL-2 induced IL-10 secretion by redirected $\mathrm{CD}^{+} \mathrm{T}$ cells. Oncoimmunology 1, 458-466 (2012)

54. Hombach, A. A., Chmielewski, M., Rappl, G. \& Abken, $\mathrm{H}$. Adoptive immunotherapy with redirected $\mathrm{T}$ cells produces $\mathrm{CCR} 7$ cells that are trapped in the periphery and benefit from combined CD28-OX40 costimulation. Hum. Gene Ther. 24, 259-269 (2013).

55. Guedan, S. et al. ICOS-based chimeric antigen receptors program bipolar $\mathrm{T}_{H} 17 / \mathrm{T}_{H} 1$ cells. Blood 124, 1070-1080 (2014)

56. Song, D. G. \& Powell, D. J. Pro-survival signaling via CD27 costimulation drives effective CAR T-cell therapy. Oncoimmunology 1, 547-549 (2012).

57. Duong, C. P. et al. Engineering T cell function using chimeric antigen receptors identified using a DNA library approach. PLOS ONE 8, e63037 (2013).

58. Wang, E. et al. Generation of potent T-cell immunotherapy for cancer using DAP12-based, multichain, chimeric immunoreceptors. Cancer Immunol. Res. 3, 815-826 (2015).

59. Brudno, J. N. \& Kochenderfer, J. N. Recent advances in CAR T-cell toxicity: mechanisms, manifestations and management. Blood Rev. 34, 45-55 (2019).

60. Lee, D. W. et al. ASTCT consensus grading for cytokin release syndrome and neurologic toxicity associated with immune effector cells. Biol. Blood Marrow Transplant. 25, 625-638 (2019).

61. Santomasso, B. D. et al. Clinical and biological correlates of neurotoxicity associated with CAR T-cell therapy in patients with B-cell acute lymphoblastic leukemia. Cancer Discov. 8, 958-971 (2018)

62. Hay, K. A. et al. Kinetics and biomarkers of severe cytokine release syndrome after CD 19 chimeric antigen receptor-modified T-cell therapy. Blood 130 2295-2306 (2017).

63. Brentjens, R. J. et al. CD19-targeted T cells rapidly induce molecular remissions in adults with chemotherapy-refractory acute lymphoblastic leukemia. Sci. Transl. Med. 5, 177ra138 (2013).

64. Milone, M. C. \& Bhoj, V. G. The pharmacology of T cell therapies. Mol. Ther. Methods Clin. Dev. 8, 210-221 (2018)

65. van der Stegen, S. J., Hamieh, M. \& Sadelain, M. The pharmacology of second-generation chimeric antigen receptors. Nat. Rev. Drug Discov. 14 499-509 (2015)

66. Salter, A. I. et al. Phosphoproteomic analysis of chimeric antigen receptor signaling reveals kinetic and quantitative differences that affect cell function. Sci. Signal. 11, eaat6753 (2018)

67. Ying, Z. et al. A safe and potent anti-CD19 CAR T cell therapy. Nat. Med. 25, 947-953 (2019)

68. Sommermeyer, D. et al. Fully human CD19-specific chimeric antigen receptors for T-cell therapy. Leukemia 31, 2191-2199 (2017).

69. Sterner, R. M. et al. GM-CSF inhibition reduces cytokine release syndrome and neuroinflammation but enhances CAR-T cell function in xenografts. Blood 133, 697-709 (2019).
70. Sachdeva, M., Duchateau, P., Depil, S., Poirot, L. \& Valton, J. Granulocyte-macrophage colony-stimulating factor inactivation in CAR T-cells prevents monocytedependent release of key cytokine release syndrom mediators. J. Biol. Chem. 294, 5430-5437 (2019).

71. Giavridis, T. et al. CAR T cell-induced cytokine release syndrome is mediated by macrophages and abated by IL-1 blockade. Nat. Med. 24, 731-738 (2018)

72. Norelli, M. et al. Monocyte-derived IL-1 and IL-6 are differentially required for cytokine-release syndrome and neurotoxicity due to CAR T cells. Nat. Med. $\mathbf{2 4}$ 739-748 (2018)

73. Staedtke, V. et al. Disruption of a self-amplifying catecholamine loop reduces cytokine release syndrome. Nature 564, 273-277 (2018)

74. Lee, Y. G. et al. Regulation of CAR T cell-mediated cytokine release syndrome-like toxicity using low molecular weight adapters. Nat. Commun. 10, 2681 (2019).

75. Rodgers, D. T. et al. Switch-mediated activation and retargeting of CAR-T cells for B-cell malignancies. Proc. Natl Acad. Sci. USA 113, E459-E468 (2016)

76. Serafini, M. et al. Characterization of CD20-transduced T lymphocytes as an alternative suicide gene therapy approach for the treatment of graft-versus-host disease. Hum. Gene Ther. 15, 63-76 (2004).

77. Griffioen, M et al Retroviral transfer of human CD20 as a suicide gene for adoptive T-cell therapy. Haematologica 94, 1316-1320 (2009)

78. Philip, B. et al. A highly compact epitope-based marker/suicide gene for easier and safer T-cell therapy. Blood 124, 1277-1287 (2014).

79. Wang, X. et al. A transgene-encoded cell surface polypeptide for selection, in vivo tracking, and ablation of engineered cells. Blood 118, 1255-1263 (2011).

80. Di Stasi, A. et al. Inducible apoptosis as a safety switch for adoptive cell therapy. N. Engl. J. Med. 365, 1673-1683 (2011)

81. Juillerat, A. et al. Modulation of chimeric antigen receptor surface expression by a small molecule switch. BMC Biotechnol. 19, 44 (2019).

82. Mestermann, $\mathrm{K}$, et al. The tyrosine kinase inhibitor dasatinib acts as a pharmacologic on/off switch for CAR T cells. Sci. Transl. Med. 11, eaau5907 (2019).

83. Kalos, M. et al. T cells with chimeric antigen receptors have potent antitumor effects and can establish memory in patients with advanced leukemia. Sci. Transl. Med. 3 , 95ra73 (2011)

84. Lamers, C. H. J. et al. Treatment of metastatic renal cell carcinoma with CAIX CAR-engineered T cells: clinical evaluation and management of on-target toxicity. Mol. Ther. 21, 904-912 (2013)

85. Morgan, R. A. et al. Case report of a serious adverse event following the administration of T cells transduced with a chimeric antigen receptor recognizing ERBB2. Mol. Ther. 18, 843-851 (2010).

86. Seidman, A. et al. Cardiac dysfunction in the trastuzumab clinical trials experience. J. Clin. Oncol. 20, 1215-1221 (2002)

87. Ahmed, N. et al. Human epidermal growth factor receptor 2 (HER2)-specific chimeric antigen receptormodified T cells for the immunotherapy of HER2positive sarcoma. J. Clin. Oncol. 33, 1688-1696 (2015).

88. Goff, S. L. et al. Pilot trial of adoptive transfer of chimeric antigen receptor-transduced $\mathrm{T}$ cells targeting EGFRvIII in patients with glioblastoma. J. Immunother 42, 126-135 (2019)

89. Thistlethwaite, F. C. et al. The clinical efficacy of firstgeneration carcinoembryonic antigen (CEACAM5)specific CAR T cells is limited by poor persistence and transient pre-conditioning-dependent respiratory toxicity. Cancer Immunol. Immunother. 66, 1425-1436 (2017).

90. Caruso, H. G., Heimberger, A. B. \& Cooper, L. J. N. Steering CAR T cells to distinguish friend from foe. Oncoimmunology 8, e1271857 (2018).

91. Liu, K. et al. Retargeted human avidin-CAR T cells for adoptive immunotherapy of EGFRvIll expressing gliomas and their evaluation via optical imaging. Oncotarget 6, 23735-23747 (2015)

92. Song, D.-G. et al. A fully human chimeric antigen receptor with potent activity against cancer cells but reduced risk for off-tumor toxicity. Oncotarget 6, 21533-21546 (2015)

93. Richman, S. A. et al. High-affinity GD2-specific CAR $T$ cells induce fatal encephalitis in a preclinical neuroblastoma model. Cancer Immunol. Res. 6 36-46 (2018)

94. Roybal, KoleT. et al. Precision tumor recognition by $T$ cells with combinatorial antigen-sensing circuits. Cell 164, 770-779 (2016).
95. Srivastava, S. et al. Logic-gated ROR1 chimeric antigen receptor expression rescues $\mathrm{T}$ cell-mediated toxicity to normal tissues and enables selective tumor targeting. Cancer Cell 35, 489-503.e8 (2019).

96. Kloss, C. C., Condomines, M., Cartellieri, M. Bachmann, M. \& Sadelain, M. Combinatorial antigen recognition with balanced signaling promotes selective tumor eradication by engineered T cells. Nat. Biotechnol. 31, 71-75 (2013).

97. Wilkie, S. et al. Dual targeting of ErbB2 and MUC1 in breast cancer using chimeric antigen receptors engineered to provide complementary signaling. J. Clin. Immunol. 32, 1059-1070 (2012).

98. Lanitis, E. et al. Chimeric antigen receptor T cells with dissociated signaling domains exhibit focused antitumor activity with reduced potential for toxicity in vivo. Cancer Immunol. Res. 1, 43-53 (2013).

99. Leen, A. M. et al. Reversal of tumor immune inhibition using a chimeric cytokine receptor. Mol. Ther. 22, 1211-1220 (2014).

100. Fedorov, V. D., Themeli, M. \& Sadelain, M. PD-1- and CTLA-4-based inhibitory chimeric antigen receptors (iCARs) divert off-target immunotherapy responses. Sci. Transl. Med. 5, 215 ra172 (2013).

101. Juillerat, A. et al. An oxygen sensitive self-decision making engineered CAR T-cell. Sci. Rep. 7, 39833 (2017).

102. Tchou, J. et al. Safety and efficacy of intratumoral injections of chimeric antigen receptor (CAR) T cells in metastatic breast cancer. Cancer Immunol. Res. 5. 1152-1161 (2017)

103. Wu, C. Y., Roybal, K. T., Puchner, E. M., Onuffer, J. $\&$ Lim, W. A. Remote control of therapeutic T cells through a small molecule-gated chimeric receptor. Science 350, aab4077 (2015).

104. Juillerat, A. et al. Design of chimeric antigen receptors with integrated controllable transient functions. Sci. Rep. 6, 18950 (2016).

105. Fisher, J. \& Anderson, J. Engineering approaches in human gamma delta $T$ cells for cancer immunotherapy. Front. Immunol. 9, 1409 (2018).

106. Fisher, J. et al. Avoidance of on-target off-tumor activation using a co-stimulation-only chimeric antigen receptor. Mol. Ther. 25, 1234-1247 (2017).

107. Kim, M. Y. et al. Genetic inactivation of CD33 in hematopoietic stem cells to enable CAR T cell immunotherapy for acute myeloid leukemia. Cell 173, 1439-1453.e19 (2018).

108. Majzner, R. G. \& Mackall, C. L. Tumor antigen escape from CAR T-cell therapy. Cancer Discov. 8, 1219-1226 (2018).

109. Rafiq, S. \& Brentjens, R. J. Tumors evading CARs - the chase is on. Nat. Med. 24, 1492-1493 (2018).

110. Pan, J. et al. CD22 CAR T-cell therapy in refractory or relapsed $B$ acute lymphoblastic leukemia. Leukemia 33, 2854-2866 (2019).

111. Shah, N. N. et al. Results of a phase I study of bispecific anti-CD 19, anti-CD20 chimeric antigen receptor (CAR) modified T cells for relapsed, refractory, non-Hodgkin lymphoma. J. Clin. Oncol. 37, 2510-2510 (2019).

112. Mahadeo, K. M. et al. Management guidelines for paediatric patients receiving chimeric antigen receptor T cell therapy. Nat. Rev. Clin. Oncol. 16, 45-63 (2019).

113. Kantarjian, $\mathrm{H}$. et al. Blinatumomab versus chemotherapy for advanced acute lymphoblastic leukemia. N. Engl. J. Med. 376, 836-847 (2017).

114. Choi, B. D. et al. CAR-T cells secreting BiTEs circumvent antigen escape without detectable toxicity. Nat. Biotechnol. 37, 1049-1058 (2019).

115. Curran, K. J. et al. Enhancing antitumor efficacy of chimeric antigen receptor $T$ cells through constitutive CD40L expression. Mol. Ther. 23, 769-778 (2015).

116. Kuhn, N. F. et al. CD40 ligand-modified chimeric antigen receptor $\mathrm{T}$ cells enhance antitumor function by eliciting an endogenous antitumor response. Cancer Cell 35, 473-488.e6 (2019).

117. Boice, M. et al. Loss of the HVEM tumor suppressor in lymphoma and restoration by modified CAR-T cells. Cell 167, 405-418.e13 (2016).

118. Busch, D. H., Fräßle, S. P., Sommermeyer, D., Buchholz, V. R. \& Riddell, S. R. Role of memory T cell subsets for adoptive immunotherapy. Semin. Immunol. 28, 28-34 (2016)

119. Morgan, M. A. \& Schambach, A. Engineering CARcells for improved function against solid tumors. Front. Immunol. 9, 2493 (2018).

120. Petersen, C. T. et al. Improving T-cell expansion and function for adoptive T-cell therapy using ex vivo treatment with PI3K $\delta$ inhibitors and VIP antagonists. Blood Adv. 2, 210-223 (2018). 
121. Turtle, C. J. et al. CD19 CAR-T cells of defined $\mathrm{CD}^{+}: \mathrm{CD} 8^{+}$composition in adult $\mathrm{B}$ cell ALL patients. J. Clin. Invest. 126, 2123-2138 (2016).

122. Turtle, C. J. et al. Immunotherapy of non-Hodgkin's lymphoma with a defined ratio of $\mathrm{CD}^{+}$and $\mathrm{CD}^{+}+$ CD19-specific chimeric antigen receptor-modified T cells. Sci. Transl. Med. 8, 355ra116 (2016).

123. Sabatino, M. et al. Generation of clinical-grade CD19-specific CAR-modified CD8 ${ }^{+}$memory stem cells for the treatment of human B-cell malignancies. Blood 128, 519-528 (2016).

124. Kochenderfer, J. N., Yu, Z., Frasheri, D., Restifo, N. P. $£$ Rosenberg, S. A. Adoptive transfer of syngeneic $T$ cells transduced with a chimeric antigen receptor that recognizes murine $C D 19$ can eradicate lymphoma and normal B cells. Blood 116, 3875-3886 (2010).

125. Boucher, J. C. et al. Mutation of the CD28 costimulatory domain confers increased CAR T cell persistence and decreased exhaustion. J. Immunol. 200, 57.28 (2018)

126. Feucht, J. et al. Calibration of CAR activation potential directs alternative $T$ cell fates and therapeutic potency. Nat. Med. 25, 82-88 (2019).

127. Zhao, Z. et al. Structural design of engineered costimulation determines tumor rejection kinetics and persistence of CAR T cells. Cancer Cell 28, 415-428 (2015).

128. Park, J. H. et al. A phase I first-in-human clinical trial of CD 19-targeted 19-28z/4-1BBL "armored" CAR $\mathrm{T}$ cells in patients with relapsed or refractory NHL and CLL including Richter's transformation. Blood 132, 224 (2018).

129. Centers for Disease Control and Prevention. United States Cancer Statistics: Data Visualizations https://www.cdc.gov/cancer/dataviz (2019).

130. Junttila, M. R. \& de Sauvage, F. J. Influence of tumour micro-environment heterogeneity on therapeutic response. Nature 501, 346-354 (2013).

131. Wing, A. et al. Improving CART-cell therapy of solid tumors with oncolytic virus-driven production of a bispecific T-cell engager. Cancer Immunol. Res. 6 , 605-616 (2018).

132. Urbanska, K. et al. A universal strategy for adoptive immunotherapy of cancer through use of a novel T-cell antigen receptor. Cancer Res. 72, 1844-1852 (2012).

133. Lohmueller, J. J., Ham, J. D., Kvorjak, M. \& Finn, O. J. mSA2 affinity-enhanced biotin-binding CAR T cells for universal tumor targeting. Oncoimmunology 7 e1368604 (2017).

134. Tamada, K. et al. Redirecting gene-modified T cells toward various cancer types using tagged antibodies. Clin. Cancer Res. 18, 6436-6445 (2012)

135. Kim, M. S. et al. Redirection of genetically engineered CAR-T cells using bifunctional small molecules. J. Am. Chem. Soc. 137, 2832-2835 (2015).

136. Ma, J. S. Y. et al. Versatile strategy for controlling the specificity and activity of engineered T cells. Proc. Nat Acad. Sci. USA 113, E450-E458 (2016).

137. Lee, Y. G. et al. Use of a single CAR T cell and severa bispecific adapters facilitates eradication of multiple antigenically different solid tumors. Cancer Res. 79, 387-396 (2019)

138. Kudo, K. et al. T lymphocytes expressing a CD 16 signaling receptor exert antibody-dependent cancer cell killing. Cancer Res. 74, 93-103 (2014).

139. Cho, J. H., Collins, J. J. \& Wong, W. W. Universal chimeric antigen receptors for multiplexed and logical control of T cell responses. Cell 173, 1426-1438.e11 (2018).

140. Beatty, G. L. et al. Mesothelin-specific chimeric antigen receptor mRNA-engineered T cells induce anti-tumor activity in solid malignancies. Cancer Immunol. Res. 2, 112-120 (2014).

141. Katz, S. C. et al. Phase I hepatic immunotherapy for metastases study of intra-arterial chimeric antige receptor-modified T-cell therapy for $\mathrm{CEA}^{+}$liver metastases. Clin. Cancer Res. 21, 3149-3159 (2015).

142. Hardaway, J. C., Prince, E., Arepally, A. \& Katz, S. C. Regional infusion of chimeric antigen receptor T cells to overcome barriers for solid tumor immunotherapy. J. Vasc. Interv. Radiol. 29, 1017-1021.e1 (2018).

143. Sridhar, P. \& Petrocca, F. Regional delivery of chimeric antigen receptor (CAR) T-cells for cancer therapy. Cancers 9, E92 (2017).

144. Nagarsheth, N., Wicha, M. S. \& Zou, W. Chemokines in the cancer microenvironment and their relevance in cancer immunotherapy. Nat. Rev. Immunol. 17 559-572 (2017)

145. Lo, A. S. et al. Harnessing the tumour-derived cytokine, CSF-1, to co-stimulate T-cell growth and activation. Mol. Immunol. 45, 1276-1287 (2008).
146. Di Stasi, A. et al. T lymphocytes coexpressing CCR4 and a chimeric antigen receptor targeting CD30 have improved homing and antitumor activity in a Hodgkin tumor model. Blood 113, 6392-6402 (2009).

147. Craddock, J. A. et al. Enhanced tumor trafficking of GD2 chimeric antigen receptor $T$ cells by expression of the chemokine receptor CCR2b. J. Immunother. 33, 780-788 (2010).

148. Moon, E. K. et al. Expression of a functional CCR2 receptor enhances tumor localization and tumor eradication by retargeted human $\mathrm{T}$ cells expressing a mesothelin-specific chimeric antibody receptor. Clin. Cancer Res. 17, 4719-4730 (2011).

149. Tran, E. et al. Immune targeting of fibroblast activation protein triggers recognition of multipotent bone marrow stromal cells and cachexia. J. Exp. Med. 210, 1125-1135 (2013).

150. Wang, L. C. et al. Targeting fibroblast activation protein in tumor stroma with chimeric antigen receptor T cells can inhibit tumor growth and augment host immunity without severe toxicity. Cancer Immunol. Res. 2 154-166 (2014)

151. Caruana, I. et al. Heparanase promotes tumor infiltration and antitumor activity of CAR-redirected T lymphocytes. Nat. Med. 21, 524-529 (2015).

152. Mardomi, A. \& Abediankenari, S. Matrix metalloproteinase 8: could it benefit the CAR-T cell therapy of solid tumors? - a- Commentary on therapeutic potential. Cancer Microenviron. 11, 93-96 (2018)

153. Ramanathan, R. K. et al. Phase IB/II randomized study of FOLFIRINOX plus pegylated recombinant human hyaluronidase versus FOLFIRINOX alone in patients with metastatic pancreatic adenocarcinoma: SWOC S1313. J. Clin. Oncol. 37, 1062-1069 (2019).

154. Hingorani, S. R. et al. HALO 202: Randomized phase II study of PEGPH20 plus Nab-paclitaxel/gemcitabine versus Nab-paclitaxel/gemcitabine in patients with untreated, metastatic pancreatic ductal adenocarcinoma. J. Clin. Oncol. 36, 359-366 (2018)

155. Postow, M. A., Callahan, M. K. \& Wolchok, J. D. Immune checkpoint blockade in cancer therapy. J. Clin. Oncol. 33, 1974-1982 (2015).

156. Moon, E. K. et al. Multifactorial T-cell hypofunction that is reversible can limit the efficacy of chimeric antigen receptor-transduced human T cells in solid tumors. Clin. Cancer Res. 20, 4262-4273 (2014).

157. Yoon, D. H., Osborn, M. J., Tolar, J. \& Kim, C. J. Incorporation of immune checkpoint blockade into chimeric antigen receptor T cells (CAR-Ts): combination or built-in CAR-T. Int. J. Mol. Sci. 19 E340 (2018)

158. Li, A. M. et al. Checkpoint inhibitors augment CD 19-directed chimeric antigen receptor (CAR) $\mathrm{T}$ cell therapy in relapsed B-cell acute lymphoblastic leukemia. Blood 132, 556 (2018).

159. Chong, E. A. et al. Phase I/II study of pembrolizumab for progressive diffuse large B cell lymphoma after anti-CD 19 directed chimeric antigen receptor modified T cell therapy. Blood 130, 4121 (2017).

160. Adusumilli P. S. et al. A phase I clinical trial of malignant pleural disease treated with regionally delivered autologous mesothelin-targeted CAR T cells: safety and efficacy - a preliminary report. Presented at the American Society of Gene and Cell 21st Annual Meeting (2018).

161. Cherkassky, L. et al. Human CAR T cells with cellintrinsic PD-1 checkpoint blockade resist tumormediated inhibition. J. Clin. Invest. 126, 3130-3144 (2016).

162. Prosser, M. E., Brown, C. E., Shami, A. F., Forman, S. J. \& Jensen, M. C. Tumor PD-L1 co-stimulates primary human $\mathrm{CD} 8^{+}$cytotoxic $\mathrm{T}$ cells modified to express a PD 1:CD28 chimeric receptor. Mol. Immunol. 51, 263-272 (2012)

163. Ankri, C., Shamalov, K., Horovitz-Fried, M., Mauer, S. $\&$ Cohen, $C$. J. Human T cells engineered to express a programmed death $1 / 28$ costimulatory retargeting molecule display enhanced antitumor activity. J. Immunol. 191, 4121-4129 (2013).

164. Kobold, S. et al. Impact of a new fusion receptor on PD-1-mediated immunosuppression in adoptive $\mathrm{T}$ cell therapy. J. Natl Cancer Inst. 107, djv1 46 (2015).

165. Liu, X. et al. A chimeric switch-receptor targeting PD1 augments the efficacy of second-generation CAR T cells in advanced solid tumors. Cancer Res. 76 1578-1590 (2016).

166. Suarez, E. R. et al. Chimeric antigen receptor T cells secreting anti-PD-L1 antibodies more effectively regress renal cell carcinoma in a humanized mouse model. Oncotarget 7, 34341-34355 (2016).
167. Rafiq, S. et al. Targeted delivery of a PD-1-blocking scFv by CAR-T cells enhances anti-tumor efficacy in vivo. Nat. Biotechnol. 36, 847-856 (2018).

168. Li, S. et al. Enhanced cancer immunotherapy by chimeric antigen receptor-modified T cells engineered to secrete checkpoint inhibitors. Clin. Cancer Res. 23 6982-6992 (2017).

169. Rupp, L. J. et al. CRISPR/Cas9-mediated PD-1 disruption enhances anti-tumor efficacy of human chimeric antigen receptor T cells. Sci. Rep. 7, 737 (2017).

170. Simon, S. \& Labarriere, N. PD-1 expression on tumorspecific T cells: friend or foe for immunotherapy? Oncoimmunology 7, e1364828 (2017).

171. Wei, J. et al. PD-1 silencing impairs the anti-tumor function of chimeric antigen receptor modified T cells by inhibiting proliferation activity. J. Immunother. Cancer 7, 209 (2019).

172. Condomines, M. et al. Tumor-targeted human T cells expressing CD28-based chimeric antigen receptors circumvent CTLA-4 inhibition. PLOS ONE 10, e0130518 (2015)

173. Newick, K. et al. Augmentation of CAR T-cell trafficking and antitumor efficacy by blocking protein kinase $A$ localization. Cancer Immunol. Res. 4, 541-551 (2016).

174. Beavis, P. A. et al. Targeting the adenosine $2 \mathrm{~A}$ receptor enhances chimeric antigen receptor $\mathrm{T}$ cell efficacy. J. Clin. Invest. 127, 929-941 (2017).

175. Siriwon, N. et al. CAR-T cells surface-engineered with drug-encapsulated nanoparticles can ameliorate intratumoral T-cell hypofunction. Cancer Immunol. Res. 6, 812-824 (2018).

176. Kloss, C. C. et al. Dominant-negative TGF- $\beta$ receptor enhances PSMA-targeted human CAR T cell proliferation and augments prostate cancer eradication. Mol. Ther 26, 1855-1866 (2018).

177. Mohammed, S. et al. Improving chimeric antigen receptor-modified $\mathrm{T}$ cell function by reversing the immunosuppressive tumor microenvironment of pancreatic cancer. Mol. Ther. 25, 249-258 (2017).

178. Wilkie, S. et al. Selective expansion of chimeric antigen receptor-targeted T-cells with potent effector function using interleukin-4. J. Biol. Chem. 285, 25538-25544 (2010).

179. Yamamoto, T. N. et al. T cells genetically engineered to overcome death signaling enhance adoptive cancer immunotherapy. J. Clin. Invest. 129, 1551-1565 (2019).

180. Xu, X., Gnanaprakasam, J. N. R., Sherman, J. \& Wang, R. A metabolism toolbox for CAR T therapy. Front. Oncol. 9, 322 (2019).

181. Geiger, R. et al. L-arginine modulates T cell metabolism and enhances survival and anti-tumor activity. Cell 167, 829-842.e13 (2016).

182. Vodnala, S. K. et al. T cell stemness and dysfunction in tumors are triggered by a common mechanism. Science 363, eaau0135 (2019).

183. Ando, T. et al. Transduction with the antioxidant enzyme catalase protects human T cells against oxidative stress. J. Immunol. 181, 8382-8390 (2008).

184. Chmielewski, M., Hombach, A. A. \& Abken, H. Of CARs and TRUCKs: chimeric antigen receptor (CAR) $T$ cells engineered with an inducible cytokine to modulate the tumor stroma. Immunol. Rev. 257 83-90 (2014).

185. Pegram, H. J. et al. Tumor-targeted T cells modified to secrete IL-12 eradicate systemic tumors without need for prior conditioning. Blood 119, 4133-414 (2012).

186. Koneru, M., Purdon, T. J., Spriggs, D., Koneru, S. $\Sigma$ Brentjens, R. J. IL-12 secreting tumor-targeted chimeric antigen receptor T cells eradicate ovarian tumors in vivo. Oncoimmunology 4, e994446 (2015).

187. Yeku, O. O., Purdon, T. J., Koneru, M., Spriggs, D. $\&$ Brentjens, R. J. Armored CAR T cells enhance antitumor efficacy and overcome the tumor microenvironment. Sci. Rep. 7, 10541 (2017).

188. Koneru, M., O'Cearbhaill, R., Pendharkar, S., Spriggs, D. R. \& Brentjens, R. J. A phase I clinical trial of adoptive $T$ cell therapy using IL-12 secreting MUC-16ecto directed chimeric antigen receptors for recurrent ovarian cancer. J. Transl. Med. 13, 102 (2015).

189. Chmielewski, M., Kopecky, C., Hombach, A. A $\&$ Abken, H. IL-12 release by engineered T cells expressing chimeric antigen receptors can effectively muster an antigen-independent macrophage response on tumor cells that have shut down tumor antigen expression. Cancer Res. 71, 5697-5706 (2011). 
190. Chinnasamy, D. et al. Local delivery of interleukin-12 using T cells targeting VEGF receptor-2 eradicates multiple vascularized tumors in mice. Clin. Cancer Res. 18, 1672-1683 (2012)

191. Leonard, J. P. et al. Effects of single-dose interleukin-12 exposure on interleukin-12-associated toxicity and interferon- $\gamma$ production. Blood 90, 2541-2548 (1997).

192. Zhang, L. et al. Tumor-infiltrating lymphocytes genetically engineered with an inducible gene encoding interleukin-12 for the immunotherapy of metastatic melanoma. Clin. Cancer Res. 21, 2278-2288 (2015).

193. Mizuguchi, H., Xu, Z., Ishii-Watabe, A., Uchida, E. \& Hayakawa, T. IRES-dependent second gene expression is significantly lower than Cap-dependent first gene expression in a bicistronic vector. Mol. Ther. 1 , 376-382 (2000).

194. Zhang, L. et al. Improving adoptive T cell therapy by targeting and controlling IL-12 expression to the tumor environment. Mol. Ther. 19, 751-759 (2011)

195. Hoyos, V. et al. Engineering CD 19-specific T lymphocytes with interleukin-15 and a suicide gene to enhance their anti-lymphoma/leukemia effects and safety. Leukemia 24, 1160-1170 (2010).

196. Krenciute, G. et al. Transgenic expression of IL15 improves antiglioma activity of IL13Ra2-CAR T cells but results in antigen loss variants. Cancer Immunol. Res. 5, 571-581 (2017)

197. Chen, Y. et al. Eradication of neuroblastoma by T cells redirected with an optimized GD2-specific chimeric antigen receptor and interleukin-15. Clin. Cancer Res. 25, 2915-2924 (2019).

198. Hurton, L. V. et al. Tethered IL-15 augments antitumo activity and promotes a stem-cell memory subset in tumor-specific T cells. Proc. Natl Acad. Sci. USA 113 E7788-E7797 (2016).

199. Nair, S. et al. Functional improvement of chimeric antigen receptor through intrinsic interleukin-15Ra signaling. Curr. Gene Ther. 19, 40-53 (2019).

200. Hsu, C. et al. Cytokine-independent growth and clonal expansion of a primary human $\mathrm{CD} 8^{+} \mathrm{T}$-cell clone following retroviral transduction with the IL-15 gene. Blood 109, 5168-5177 (2007).

201. Fehniger, T. A. et al. Fatal leukemia in interleukin 15 transgenic mice follows early expansions in natural killer and memory phenotype CD8 ${ }^{+}$T cells. J. Exp. Med. 193, 219-231 (2001)

202. Conlon, K C . et al. Redistribution, hyperproliferation, activation of natural killer cells and CD8 T cells, and cytokine production during first-in-human clinical trial of recombinant human interleukin-15 in patients with cancer. J. Clin. Oncol. 33, 74-82 (2015).

203. Tang, L. et al. Enhancing T cell therapy through TCR-signaling-responsive nanoparticle drug delivery. Nat Biotechnol. 36, 707 (2018).

204. Carroll, R. G. et al. Distinct effects of IL-18 on the engraftment and function of human effector CD8 T cells and regulatory T cells. PLOS ONE 3, e3289 (2008).

205. Avanzi, M. P. et al. Engineered tumor-targeted T cells mediate enhanced anti-tumor efficacy both directly and through activation of the endogenous immune system. Cell Rep. 23, 2130-2141 (2018).

206. Chmielewski, M. \& Abken, H. CAR T cells releasing IL-18 convert to T-Bet ${ }^{\text {high }}$ FoxO $1^{\text {low }}$ effectors that exhibit augmented activity against advanced solid tumors. Cell Rep. 21, 3205-3219 (2017).

207. Hu, B. et al. Augmentation of antitumor immunity by human and mouse CAR T cells secreting IL-18. Cell Rep. 20, 3025-3033 (2017)

208. Robertson, M. J. et al. Clinical and biological effects of recombinant human interleukin-18 administered by intravenous infusion to patients with advanced cancer. Clin Cancer Res. 12, 4265-4273 (2006).

209. Sedimbi, S. K., Hagglof, T. \& Karlsson, M. C. IL-18 in inflammatory and autoimmune disease. Cell. Mol. Life Sci. 70, 4795-4808 (2013).

210. Vidal-Vanaclocha, F. et al. Clinical and experimental approaches to the pathophysiology of interleukin-18 in cancer progression. Cancer Metastasis Rev. 25, 417-434 (2006)

211. Adachi, K. et al. IL-7 and CCL19 expression in CAR-T cells improves immune cell infiltration and CAR-T cell survival in the tumor. Nat. Biotechnol. 36, 346-351 (2018)

212. Chen, Y. $\&$ Lu, B. Guided delivery of the "alarming" cytokine IL-33 to tumor by chimeric antigen receptor T cells. J. Immunol. 198, 204.223 (2017).
213. Li, X. D., A., Lopez, A., Purdon, T. \& Brentjens, R. Augmenting CAR T cell mediated antitumor efficacy through genetic modification to secrete a novel cytokine IL-36 $\gamma$. Mol. Ther. 27, 432-433 (2019).

214. Singh, N., Perazzelli, J., Grupp, S. A. \& Barrett, D. M. Early memory phenotypes drive $\mathrm{T}$ cell proliferation in patients with pediatric malignancies. Sci. Transl Med. 8, 320 ra323 (2016)

215. Das, R. K., Vernau, L., Grupp, S. A. \& Barrett, D. M. Naïve T-cell deficits at diagnosis and after chemotherapy impair cell therapy potential in pediatric cancers. Cancer Discov 9 492-499 (2019).

216. Elavia, N. et al. Effects of starting cellular materia composition on chimeric antigen receptor T-cel expansion and characteristics. Transfusion $\mathbf{5 9}$ 1755-1764 (2019).

217. Schietinger, A. et al. Tumor-specific T cell dysfunction is a dynamic antigen-driven differentiation program initiated early during tumorigenesis. Immunity 45, 389-401 (2016)

218. Totterman, T., Carlsson, M., Simonsson, B. Bengtsson, M. \& Nilsson, K. T-cell activation and subset patterns are altered in B-CLL and correlate with the stage of the disease. Blood 74, 786-792 (1989).

219. Fraietta, J. A. et al. Determinants of response and resistance to $C D 19$ chimeric antigen receptor (CAR) $\mathrm{T}$ cell therapy of chronic lymphocytic leukemia. Nat. Med. 24, 563-571 (2018)

220. Philip, M. et al. Chromatin states define tumourspecific $T$ cell dysfunction and reprogramming. Nature 545, 452 (2017).

221. Kochenderfer, J. N. et al. Donor-derived CD19-targeted $T$ cells cause regression of malignancy persisting after allogeneic hematopoietic stem cell transplantation. Blood 122, 4129-4139 (2013).

222. Kebriaei, P. et al. Phase I trials using Sleeping Beauty to generate CD19-specific CAR T cells. J. Clin. Invest. 126, 3363-3376 (2016)

223. Brudno, J. N. et al. Allogeneic T cells that express an anti-CD19 chimeric antigen receptor induce remissions of B-cell malignancies that progress after allogeneic hematopoietic stem-cell transplantation without causing graft-versus-host disease. J. Clin. Oncol. 34, 1112-1121 (2016)

224. Ghosh, A. et al. Donor CD19 CAR T cells exert potent graft-versus-lymphoma activity with diminished graft-versus-host activity. Nat. Med. 23, 242 (2017).

225. Cruz, C. R. Y. et al. Infusion of donor-derived CD19redirected virus-specific $\mathrm{T}$ cells for $\mathrm{B}$-cell malignancies relapsed after allogeneic stem cell transplant: a phase 1 study. Blood 122, 2965-2973 (2013).

226. Rotolo, R. et al. CAR-based strategies beyond T lymphocytes: integrative opportunities for cancer adoptive immunotherapy. Int. J. Mol. Sci. 20, 2839 (2019).

227. Hu W., Wang G., Huang D. Sui, M \& Xu, Y. Cancer immunotherapy based on natural killer cells: current progress and new opportunities. Front. Immunol. 10, 1205 (2019)

228. Osborn, M. J. et al. Evaluation of TCR gene editing achieved by TALENs, CRISPR/Cas9, and megaTAL nucleases. Mol. Ther. 24, 570-581 (2016).

229. Torikai, H. et al. A foundation for universal T-cell based immunotherapy: T cells engineered to express a CD19-specific chimeric-antigen-receptor and eliminate expression of endogenous TCR. Blood 119, 5697-5705 (2012)

230. Torikai, H. et al. Toward eliminating HLA class expression to generate universal cells from allogeneic donors. Blood 122, 1341-1349 (2013).

231. Ren, J. et al. Multiplex genome editing to generate universal CAR T cells resistant to PD1 inhibition. Clin. Cancer Res. 23, 2255-2266 (2017).

232. Poirot, L. et al. Multiplex genome-edited T-cell manufacturing platform for "off-the-shelf" adoptive T-cell immunotherapies. Cancer Res. 75, 3853-3864 (2015).

233. Qasim, W. et al. Molecular remission of infant B-ALL after infusion of universal TALEN gene-edited CAR T cells. Sci. Transl. Med. 9, eaaj2013 (2017)

234. Georgiadis, C. et al. Long terminal repeat CRISPRCAR-coupled "universal" $T$ cells mediate potent anti-leukemic effects. Mol. Ther. 26, 1215-1227 (2018).

235. Liu, X. et al. CRISPR-Cas9-mediated multiplex gene editing in CAR-T cells. Cell Res. 27, 154-157 (2017).
236. Ren, J. et al. A versatile system for rapid multiplex genome-edited CAR T cell generation. Oncotarget 8, 17002-17011 (2017).

237. Barnett, B. Manufacture of allogeneic "Universal Donor" CAR-T therapies using piggyBac ${ }^{\text {Th }}$ and Cas-CLOVER in CAR-TCR Summit, Boston MA https:// poseida.com/wp-content/uploads/2018-CAR-TCRSummit.P-BCMA-ALLO1-1.pdf (2018).

238. Eyquem, J. et al. Targeting a CAR to the TRAC locus with CRISPR/Cas9 enhances tumour rejection. Nature 543, 113 (2017)

239. MacLeod, D. T. et al. Integration of a CD19 CAR into the TCR alpha chain locus streamlines production of allogeneic gene-edited CAR T cells. Mol. Ther. 25 949-961 (2017)

240. Kamiya, T., Wong, D., Png, Y. T. \& Campana, D. A novel method to generate T-cell receptor-deficient chimeric antigen receptor T cells. Blood Adv. 2, 517-528 (2018).

241. Graham, C., Jozwik, A., Pepper, A. \& Benjamin, R. Allogeneic CAR-T cells: more than ease of access? Cells 7, 155 (2018)

242. Iriguchi, S. \& Kaneko, S. Toward the development of true "off-the-shelf" synthetic T-cell immunotherapy. Cancer Sci. 110, 16-22 (2019).

243. Zakrzewski, J. L. et al. Tumor immunotherapy across MHC barriers using allogeneic T-cell precursors. Nat. Biotechnol. 26, 453 (2008).

244. Papapetrou, E. P., Kovalovsky, D., Beloeil, L., Sant'angelo, D. \& Sadelain, M. Harnessing endogenous miR-181 a to segregate transgenic antigen receptor expression in developing versus post-thymic $T$ cells in murine hematopoietic chimeras. J. Clin. Invest. 119, 157-168 (2009).

245. Themeli, M. et al. Generation of tumor-targeted human T lymphocytes from induced pluripotent stem cells for cancer therapy. Nat. Biotechnol. 31, 928 (2013).

246. van der Stegen, S. et al. Generation of clonal antigen specific CD $8 \alpha \beta^{+}$cytotoxic T lymphocytes from renewable pluripotent stem cells for off-the-shelf $\mathrm{T}$ cell therapeutics. Blood 130 (Suppl. 1), 163 (2017).

247. Clarke, R. L. et al. Abstract LB-108: Generation of offthe-shelf TCR-less CAR-targeted cytotoxic T cells from renewable pluripotent cells for cancer immunotherapy. Cancer Res. 78, LB-108 (2018).

248. Fraietta, J. A. et al. Disruption of TET2 promotes the therapeutic efficacy of CD19-targeted T cells. Nature 558, 307-312 (2018)

249. Ruella, M. et al. Induction of resistance to chimeric antigen receptor $\mathrm{T}$ cell therapy by transduction of a single leukemic B cell. Nat. Med. 24, 1499-1503 (2018).

250. Monjezi, R. et al. Enhanced CAR T-cell engineering using non-viral Sleeping Beauty transposition from minicircle vectors. Leukemia 31, 186-194 (2017).

\section{Acknowledgements}

The authors thank the following sources of financial support: US NIH (grants 5 P01 CA190174-03 and 5 P50 CA192937-02 to R.J.B. and T32-CA009512 to C.S.H), The Annual Terry Fox Run for Cancer Research (New York, NY, USA) organized by the Canada Club of New York (R.J.B.), Kate's Team (R.J.B.), the Carson Family Charitable Trust (R J B), Mr William H. Goodwin and Mrs Alice Goodwin and their Commonwealth Foundation for Cancer Research (R.J.B.), the Center for Experimental Therapeutics of Memorial Sloan Kettering Cancer Center (R.J.B.), the Lacher Lymphoma Foundation (C.S.H), and the Druckenmiller Center for Lung Cancer Research (C.S.H).

\section{Author contributions}

All authors contributed equally to all aspects of the article.

\section{Competing interests}

R.J.B. receives royalties and grant support from JUNO Therapeutics and is a consultant for JUNO Therapeutics/ Celgene. The other authors declare no competing interests.

\section{Peer review information}

Nature Reviews Clinical Oncology thanks the three, anonymous, reviewers for their contribution to the peer review of this work.

\section{Publisher's note}

Springer Nature remains neutral with regard to jurisdictional claims in published maps and institutional affiliations.

C) Springer Nature Limited 2019 